SISSA 204/94/EP

hep-th/9412181

\title{
THE RENORMALIZATION GROUP FLOW OF THE DILATON POTENTIAL
}

\author{
R. Floreanini * \\ Istituto Nazionale di Fisica Nucleare, Sezione di Trieste \\ Dipartimento di Fisica Teorica, Università di Trieste \\ Strada Costiera 11, 34014 Trieste, Italy \\ R. Percacci ${ }^{* *}$ \\ International School for Advanced Studies, Trieste, Italy \\ via Beirut 4, 34014 Trieste, Italy \\ and \\ Istituto Nazionale di Fisica Nucleare, Sezione di Trieste
}

\begin{abstract}
We consider a scalar-metric gauge theory of gravity with independent metric, connection and dilaton. The role of the dilaton is to provide the scale of all masses, via its vacuum expectation value. In this theory, we study the renormalization group flow of the dilaton potential, taking into account threshold effects at the Planck scale. Due to the running of the VEV of the dilaton all particles that would naively seem to have masses larger than Planck's mass, may actually not propagate. This could solve the problem of unitarity in these theories.
\end{abstract}

\footnotetext{
* florean@ts.infn.it

** percacci@tsmi19.sissa.it
} 


\section{Introduction}

The general ideas that go under the name of "effective field theory" are playing an increasingly important role in elementary particle physics. The variety of physical phenomena is divided into energy ranges, whose boundaries usually coincide with the masses of various particles. In each range one has an effective field theory. Very often the theories describing neighbouring energy ranges are of the same type, the only difference being that one particle which is present in the higher energy range has been "integrated out" in the lower energy range. In other cases, however, the description of the physics in one energy range is quite different from that in the next energy range. It is clearly desirable to relate all these descriptions, but this has not always been possible so far.

It is widely believed that Einstein's General Relativity is also an effective theory, valid for energies lower than Planck's energy [1,2]. This does not mean that it can only be treated classically; it only means that any quantum calculation in Einstein's theory will have a natural cutoff at the Planck scale. From this point of view, the nonrenormalizability of Einstein's theory is not a problem.

Just below Planck's energy corrections due to higher derivative terms are expected to appear. The most general action with at most four derivatives is

$$
S\left(g_{\mu \nu}\right)=\int d^{4} x \sqrt{\operatorname{det} g}\left[\Lambda+\frac{1}{16 \pi G} R+\alpha_{1} R_{\mu \nu \rho \sigma} R^{\mu \nu \rho \sigma}+\alpha_{2} R_{\mu \nu} R^{\mu \nu}+\alpha_{3} R^{2}\right]
$$

where $R_{\mu \nu}{ }^{\rho} \sigma$ denotes the curvature tensor constructed with the metric $g_{\mu \nu}$. As emphasized in [1] the gravitational action (1.1) can be treated as a quantum field theory using the rules of "chiral perturbation theory", which were devised in the context of a theory of mesons [3]. If regarded as "fundamental", the theory (1.1) is renormalizable [4,5], but another problem appears: the terms quadratic in curvature contain higher derivatives of the metric and therefore violate unitarity. Again, this is not a problem in the "effective theory" picture: the ghosts have masses of the order of Planck's mass, and therefore will never be excited if one remains at lower energies. At energies exceeding the Planck energy, some "new physics" is expected to appear.

As mentioned above, there is no compelling reason to believe that the theory describing the physics above the Planck energy will be of the same type of the theory (1.1): it may be described by a completely different set of degrees of freedom. Nevertheless, in this paper we will conservatively assume that the "new physics" can still be described by a field theory. Motivated by the success of the gauge principle in explaining all other known forces of nature we consider a gauge theory of gravity, with independent metric and connection. We will assume that the action is at most quadratic in curvature and torsion. These theories have a long history [6]; from a particle physicist's point of view their most attractive feature is perhaps that they present a gravitational analogue of the Higgs phenomenon [7]: the vierbein behaves like a Higgs field and when it acquires a nonvanishing, constant, vacuum expectation value, its kinetic term (torsion squared) becomes a mass term for the connection. The mass is obviously of the order of Planck's mass, so below Planck's energy the connection degrees of freedom cannot be excited. Yet the connection itself does not

vanish: it becomes the Levi-Civita connection, whose components in a coordinate frame are the Christoffel symbols. In this way the theory we will consider gives rise naturally to 
the action (1.1) as an effective theory below the Planck scale. The descriptions of physics above and below Planck's energy are easy to relate in this case. The occurrence of the Higgs phenomenon is often related to unification, and indeed the theory can be generalized so as to unify gravity with all other interactions (assuming that these are already put together in a GUT). The vierbein then appears as the order parameter that breaks the symmetry between gravity and the rest [7]. This is a true unification, in the sense in which the word is used in particle physics, and does not require higher dimensional spacetimes.

The problem with these gauge theories of gravity is that there seems to be no one of them which is at the same time unitary and renormalizable. This is the same dilemma that one faces in (1.1): without the higher derivative terms the theory is not renormalizable, and with them it is not unitary. However, this time the problem cannot be avoided because we are not aware of any higher mass scale which might act as a cutoff and allow these theories to be regarded as effective field theories. Any theory that purports to describe physics above the Planck scale had better be consistent on its own.

Our point of view is that we do not know enough on these theories to draw any conclusion. The reason is, obviously, that they are very complicated. In the general case we only have a tree level analysis of their propagators [8]. A more rigorous study of the spectrum would be desirable, but for the moment has been done only for Einstein's theory [9]. It is quite possible that the quantum propagators are very different from what they seem to be at the tree level. For example, some degrees of freedom may be confined. To the best of our knowledge, no one has performed any quantum calculation so far.

Since the ghosts and/or tachyons would have masses of the order of Planck's mass, the issue of unitarity could be resolved in a rather drastic way if particles with such masses were forbidden to propagate. In this paper we elaborate on our earlier proposal for a mechanism in which this could happen. There are two main ingredients in this proposal: the dilaton, and the renormalization group. The dilaton is a scalar field, coupled to the metric, connection and to other matter fields in such a way that all masses are equal to its vacuum expectation value (VEV), multiplied by some dimensionless coupling constant. This field is closely associated to the (quantum mechanical) breaking of Weyl invariance in a manner which has been discussed in detail in $[10,11]$ and is reviewed in Appendix A. In the present work, we compute the renormalization group flow of the dilaton potential. In particular, we are interested in the running of the VEV of the dilaton, since this gives the dominant contribution to the running of the masses. (Dimensionless coupling constants are expected to run only logarithmically.) The tentative conclusion of our analysis will be that the position of the pole of a particle with mass comparable or larger than Planck's mass can be shifted to exponentially large energies. The sole remnant at lower energy would be the graviton. (In the generalized gauge theory of gravity alluded to above, one would also have an unbroken GUT sector).

This paper will be organized as follows: in Section 2 we will describe in detail the gauge theory of gravity that we are going to consider. In Section 3 we discuss the linearization of the theory around flat space and define the effective potential for the dilaton. In section 4 we define the average effective potential. In section 5 we write the renormalization group equations for the average effective potential and study their solutions. In Section 6 we draw our conclusions. 


\section{Lagrangians}

We consider a theory of gravity with independent metric $g_{\mu \nu}$, connection $\Gamma_{\lambda}{ }^{\mu}{ }_{\nu}$ and dilaton $\rho$. The connection is required to be metric:

$$
\partial_{\lambda} g_{\mu \nu}-\Gamma_{\lambda}{ }^{\tau}{ }_{\mu} g_{\tau \nu}-\Gamma_{\lambda}^{\tau}{ }_{\nu} g_{\mu \tau}=0,
$$

but can have nonvanishing torsion $T_{\lambda}{ }_{\nu}{ }_{\nu}=\Gamma_{\lambda}{ }^{\mu}{ }_{\nu}-\Gamma_{\nu}{ }^{\mu}{ }_{\lambda}$. The curvature of the connection will be denoted $R_{\mu \nu}{ }^{\rho} \sigma=\partial_{\mu} \Gamma_{\nu}{ }^{\rho}{ }_{\sigma}-\partial_{\nu} \Gamma_{\mu}{ }^{\rho} \sigma+\Gamma_{\mu}{ }^{\rho}{ }_{\tau} \Gamma_{\nu}{ }^{\tau} \sigma-\Gamma_{\nu}{ }^{\rho}{ }_{\tau} \Gamma_{\mu}{ }^{\tau} \sigma$. It is antisymmetric in $\mu, \nu$, and using (2.1) one can show that $R_{\mu \nu \alpha \beta}=g_{\alpha \rho} R_{\mu \nu}{ }^{\rho} \beta$ is also antisymmetric in $\alpha$, $\beta$. It has no other symmetry property.

The most general diffeomorphism invariant action which is at most quadratic in the derivatives of the fields is

$$
\begin{aligned}
S(g, \Gamma, \rho)=\int d^{4} x & \sqrt{\operatorname{det} g}\left[\frac{1}{2} b_{0} \partial_{\mu} \rho \partial^{\mu} \rho+2 b_{1} \rho \partial_{\mu} \rho T^{\mu}+V(\rho)+g_{0} \rho^{2} R\right. \\
& +a_{1} \rho^{2} T_{\mu \nu \rho} T^{\mu \nu \rho}+a_{2} \rho^{2} T_{\mu \nu \rho} T^{\mu \rho \nu}+a_{3} \rho^{2} T^{\rho} T_{\rho} \\
+g_{1} R_{\mu \nu \rho \sigma} R^{\mu \nu \rho \sigma}+ & \left.g_{2} R_{\mu \nu \rho \sigma} R^{\mu \rho \nu \sigma}+g_{3} R_{\mu \nu \rho \sigma} R^{\rho \sigma \mu \nu}+g_{4} R_{\mu \nu} R^{\mu \nu}+g_{5} R_{\mu \nu} R^{\nu \mu}+g_{6} R^{2}\right]
\end{aligned}
$$

where $R_{\mu \nu}=R_{\rho \mu}{ }^{\rho}{ }_{\nu}, R=g^{\mu \nu} R_{\mu \nu}$ and $T_{\nu}=T_{\nu}{ }_{\mu}{ }_{\mu}$. Indices are raised and lowered with $g$. The couplings of the dilaton can be understood as due to the quantum mechanical breaking of Weyl symmetry in a classically Weyl invariant theory. This point is discussed in Appendix A, but is not necessary for what follows.

There are some special choices of coefficients that should be pointed out since they will be useful later. The first of these can be expressed by the identity

$$
\begin{aligned}
\frac{1}{768 \pi^{2}} \int d^{4} x & \sqrt{\operatorname{det} g}\left[R_{\mu \nu \rho \sigma} R^{\rho \sigma \mu \nu}-4 R_{\mu \nu} R^{\nu \mu}+R^{2}\right] \\
& =\frac{1}{128 \pi^{2}} \int d^{4} x \frac{1}{\sqrt{\operatorname{det} g}} \varepsilon^{\alpha \beta \gamma \delta} \varepsilon^{\mu \nu \rho \sigma} R_{\alpha \beta \mu \nu} R_{\gamma \delta \rho \sigma}=\chi,
\end{aligned}
$$

$\left(\varepsilon^{1234}=1\right)$ which is an integral representation of the Euler invariant $\chi$. With this choice of action, the theory is topological. Another useful identity is

$$
R=R(g)+\frac{1}{4} T_{\mu \nu \rho} T^{\mu \nu \rho}+\frac{1}{2} T_{\mu \nu \rho} T^{\mu \rho \nu}-T^{\rho} T_{\rho}+2 \nabla_{\mu} T^{\mu},
$$

where $R(g)$ denotes the Ricci scalar of the metric $g_{\mu \nu}$ and $\nabla_{\mu}$ is the covariant derivative with respect to the Levi-Civita connection. Using this formula one can replace $R$ by $R(g)$ in (2.2) at the cost of redefining the constants $a_{i}$ and $b_{1}$. In particular, if we choose the only nonzero coefficients to be $b_{0}, g_{0}, a_{1}=-\frac{1}{4} g_{0}, a_{2}=-\frac{1}{2} g_{0}, a_{3}=g_{0}$ and $b_{1}=2 g_{0},(2.2)$ becomes the action for a nonminimally coupled scalar field:

$$
\int d^{4} x \sqrt{\operatorname{det} g}\left[\frac{1}{2} b_{0} \partial_{\mu} \rho \partial^{\mu} \rho+g_{0} R(g) \rho^{2}\right] .
$$


For $\rho=$ constant, this is Einstein's action.

Finally, we mention two alternative ways of writing the torsion squared and curvature squared terms. In discussing the linearized theory it is useful to write them in the more compact form

$$
G^{\mu \nu \alpha \beta \rho \sigma \gamma \delta} R_{\mu \nu \alpha \beta} R_{\rho \sigma \gamma \delta}+\rho^{2} H^{\mu \alpha \nu \rho \beta \sigma} T_{\mu \alpha \nu} T_{\rho \beta \sigma}
$$

where

$$
\begin{aligned}
G^{\mu \nu \alpha \beta \rho \sigma \gamma \delta}=\operatorname{SYMM}\{ & g_{1} g^{\mu \rho} g^{\nu \sigma} g^{\alpha \gamma} g^{\beta \delta}+g_{2} g^{\mu \rho} g^{\gamma \nu} g^{\alpha \sigma} g^{\beta \delta}+g_{3} g^{\gamma \mu} g^{\delta \nu} g^{\alpha \rho} g^{\beta \sigma} \\
& \left.+g^{\alpha \mu} g^{\gamma \rho}\left(g_{4} g^{\nu \sigma} g^{\beta \delta}+g_{5} g^{\delta \nu} g^{\beta \sigma}+g_{6} g^{\nu \beta} g^{\sigma \delta}\right)\right\} \\
H^{\mu \alpha \nu \rho \beta \sigma}=\operatorname{SYMM}\{ & \left.g^{\mu \rho}\left(a_{1} g^{\alpha \beta} g^{\nu \sigma}+a_{2} g^{\alpha \sigma} g^{\beta \nu}+a_{3} g^{\alpha \nu} g^{\beta \sigma}\right)\right\}
\end{aligned}
$$

The prefix "SYMM" indicates that one has to take the proper combinations so that $G$ is antisymmetric in $(\mu, \nu),(\rho, \sigma),(\alpha, \beta),(\gamma, \delta)$ and symmetric under the simultaneous interchange of $\mu \nu \alpha \beta$ and $\rho \sigma \gamma \delta$ and similarly $H$ is antisymmetric in $(\mu, \nu),(\rho, \sigma)$ and symmetric under the simultaneous interchange of $\mu \alpha \nu$ and $\rho \beta \sigma$.

The other way of writing the action is based on the decomposition of the torsion and curvature tensors into irreducible parts with respect to the Lorentz group. This is discussed in Appendix B.

\section{Linearization}

In this section we set up the formalism for computing, at one loop, the Euclidean effective action $S_{\text {eff }}(g, \Gamma, \rho)$. We will not ultimately do the calculation (this was done for example in [11]), but what we describe here is a preliminary material for the definition of the average effective action, that will be given in the next section. We will restrict ourselves to a flat background

$$
g_{\mu \nu}=\delta_{\mu \nu}, \quad \Gamma_{\lambda}{ }^{\mu}{ }_{\nu}=0, \quad \rho=\text { constant } .
$$

In this case we can write $S_{\text {eff }}(g, \Gamma, \rho)=\int d^{4} x V_{\text {eff }}(\rho)$, where $V_{\text {eff }}$ is the effective potential for the dilaton. The first step in this calculation is to linearize the action around the background (3.1). We define the fluctuation fields $\delta \Gamma_{\lambda}{ }^{\mu}{ }_{\nu}, \delta g_{\mu \nu}$ and $\delta \rho=\sigma$ as the deviations of $\Gamma, g$ and $\rho$ from their background values (3.1). In principle there is a total of $64+10+1$ fields. However, the condition (2.1) implies that

$$
\partial_{\lambda} \delta g_{\mu \nu}-\delta \Gamma_{\lambda \mu \nu}-\delta \Gamma_{\lambda \nu \mu}=0
$$

(From now on indices are raised and lowered with $\delta_{\mu \nu}$.) These are 40 constraints, reducing the number of independent fields to 35.

We define $\omega_{\lambda \mu \nu}=\delta \Gamma_{\lambda[\mu \nu]}$ and $\varphi_{\mu \nu}=\frac{1}{2} \rho \delta g_{\mu \nu}$. The rescaling of $\delta g_{\mu \nu}$ is convenient for dimensional reasons: with this definition all the fluctuations have dimension of mass. This redefinition is legitimate here since we are assuming $\rho$ to be constant; it is related to the choice of functional measure in the path integral. We will see later that it does not have any effect on the renormalization group equations. After having written out the linearized action in terms of $\delta \Gamma, \delta g$ and $\sigma$, one can use (3.2) to eliminate the symmetric part of $\delta \Gamma$ in 
favor of $\varphi: \delta \Gamma_{\lambda(\mu \nu)}=\frac{1}{\rho} \partial_{\lambda} \varphi_{\mu \nu}$. At this point the linearized Euclidean action is a quadratic form which can be written, after Fourier transforming (we use $\partial_{\mu} \rightarrow i q_{\mu}$ )

$$
S^{(2)}(\varphi, \omega, \sigma ; \rho)=\frac{1}{2} \int d^{4} q \sum_{A, B} \Phi_{A}(q) \cdot \mathcal{O}_{[A B]} \cdot \Phi_{B}(-q)
$$

where the indices $A, B$ label the three types of fields $\Phi_{1}=\omega, \Phi_{2}=\varphi$ and $\Phi_{3}=\sigma$ and the dots stand for contraction over the tensor indices. When written out explicitly in terms of the components of the fields, $\mathcal{O}$ is a $35 \times 35$ matrix. The components of this matrix are listed below:

$$
\begin{aligned}
& \mathcal{O}_{[\omega \omega] \mu \alpha \beta^{\rho \gamma \delta}}=8 G_{\mu}{ }^{\nu}{ }_{\alpha \beta}{ }^{\rho \sigma \gamma \delta} q_{\nu} q_{\sigma}+8 H_{\mu \alpha \beta}{ }^{\rho \gamma \delta} \rho^{2}+2 g_{0} \rho^{2} \delta_{\beta}^{\gamma}\left(\delta_{\alpha \mu} \delta^{\delta \rho}-\delta_{\alpha}^{\rho} \delta_{\mu}^{\delta}\right) \\
& \mathcal{O}_{[\varphi \omega] \alpha \mu}{ }^{\rho \gamma \delta}=-8 i \rho H^{\sigma}{ }_{\alpha \mu}{ }^{\rho \gamma \delta} q_{\sigma}+2 i g_{0} \rho\left(\delta_{\alpha \mu} \delta^{\delta \rho} q^{\gamma}-\delta_{\mu}^{\delta} \delta_{\alpha}^{\rho} q^{\gamma}+\delta_{\mu}^{\delta} \delta^{\gamma \rho} q_{\alpha}\right) \\
& \mathcal{O}_{[\varphi \varphi] \alpha \mu}{ }^{\beta \rho}=8 H_{\mu \alpha}{ }^{\nu \rho \beta \sigma} q_{\nu} q_{\sigma}+\frac{V}{\rho^{2}}\left(\delta_{\alpha \mu} \delta^{\beta \nu}-2 \delta_{\alpha}^{\rho} \delta_{\mu}^{\beta}\right) \\
& \mathcal{O}_{[\sigma \omega]}{ }^{\rho \gamma \delta}=2 i \rho\left(b_{1}-2 g_{0}\right) \delta^{\rho \gamma} q^{\delta} \\
& \mathcal{O}_{[\sigma \varphi]}{ }^{\mu \nu}=2 b_{1}\left(\delta^{\mu \nu} q^{2}-q^{\mu} q^{\nu}\right)+\frac{1}{\rho} \frac{d V}{d \rho} \delta^{\mu \nu} \\
& \mathcal{O}_{[\sigma \sigma]}=b_{0} q^{2}+\frac{d^{2} V}{d \rho^{2}}
\end{aligned}
$$

For the purpose of clarity we have omitted to indicate explicitly symmetrizations and antisymmetrizations on the r.h.s. (for example (3.4b) should be symmetrized in $\alpha, \mu$ and antisymmetrized in $\gamma, \delta)$. When $V=0$, this linearized action is invariant under linearized gauge transformations. Let $x^{\prime \mu}=x^{\mu}-v^{\mu}$ be an infinitesimal coordinate transformation. The variations of the fields are

$$
\delta \Gamma_{\lambda}^{\mu}{ }_{\nu}=\partial_{\lambda} \partial_{\nu} v^{\mu}, \quad \delta g_{\mu \nu}=\partial_{\mu} v_{\nu}+\partial_{\nu} v_{\mu}, \quad \delta \rho=0
$$

There follows that the fields

$$
\omega_{\lambda \mu \nu}=\frac{1}{2} q_{\lambda}\left(q_{\mu} v_{\nu}-q_{\nu} v_{\mu}\right), \quad \varphi_{\mu \nu}=\frac{i}{2} \rho\left(q_{\mu} v_{\nu}+q_{\nu} v_{\mu}\right), \quad \sigma=0
$$

are null vectors for the operator $\mathcal{O}$. This can be verified by explicit calculations. To make $\mathcal{O}$ invertible, we add to the linearized action the following gauge-fixing term:

$$
S_{\mathrm{GF}}=\frac{1}{2 \alpha} \int d^{4} x \partial_{\mu} \varphi^{\mu \nu} \partial^{\rho} \varphi_{\rho \nu} .
$$

The ghost contribution has the form

$$
S_{\text {ghost }}(\bar{d}, d)=\frac{1}{2} \int d^{4} q \bar{d}^{\mu} \mathcal{O}_{[d d]_{\mu}}{ }^{\nu} d_{\nu}
$$


where, $\bar{d}, d$, are anticommuting ghost fields and

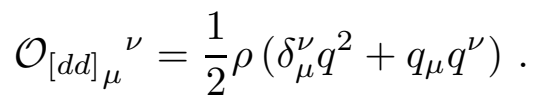

Apart from the overall factor $\rho$, which can be eliminated by a redefinition of the measure and is irrelevant, this operator is field-independent. It can be neglected in what follows.

To compute the one-loop effective action one now needs to calculate the functional determinant of the operator $\mathcal{O}$ appearing in the previous formulas. The determinant of $\mathcal{O}$ on the 35 dimensional space spanned by the fields is very hard to compute as it stands. One can partially diagonalize these operators in blocks corresponding to spin and parity. This is because $\mathcal{O}$ is a Lorentz covariant wave operator and therefore does not mix fields with different spin and parity. There are two modes with spin-parity $2^{+}$, coming from $\omega$ and $\varphi$, one $2^{-}$mode from $\omega$, two $1^{+}$modes coming from $\omega$, three $1^{-}$modes, two from $\omega$ and one from $\varphi$, four $0^{+}$of which one comes from $\omega$, two from $\varphi$ and one from $\sigma$, and finally one $0^{-}$mode from $\omega$. One counts indeed $2 \times 5+1 \times 5+2 \times 3+3 \times 3+4 \times 1+1 \times 1=35$.

The total linearized quadratic action, including the gauge-fixing, ghost and potential terms, can be rewritten as

$$
S^{(2)}=\frac{1}{2} \int d^{4} q \Phi_{A}(-q) \cdot a_{i j}^{A B}\left(J^{\mathcal{P}}\right) P_{i j}^{A B}\left(J^{\mathcal{P}}\right) \cdot \Phi_{B}(q)
$$

where $P_{i j}^{A B}\left(J^{\mathcal{P}}\right)$ are spin projection operators $[12,8,10]$ that we list in Appendix $\mathrm{C}$, and $a_{i j}^{A B}\left(J^{\mathcal{P}}\right)$ are coefficient matrices, representing the inverse propagators of each set of fields with definite spin and parity. For $V=0$ these matrices are given by

$$
\begin{aligned}
& a\left(2^{+}\right)=\left[\begin{array}{cc}
G_{1} q^{2}+B_{1} \rho^{2} & -i \sqrt{2} B_{1}|q| \rho \\
i \sqrt{2} B_{1}|q| \rho & B_{2} q^{2}
\end{array}\right], \\
& a\left(2^{-}\right)=G_{2} q^{2}+B_{1} \rho^{2}, \\
& a\left(1^{+}\right)=\left[\begin{array}{cc}
G_{3} q^{2}+B_{3} \rho^{2} & -\sqrt{2} B_{4} \rho^{2} \\
-\sqrt{2} B_{4} \rho^{2} & B_{5} \rho^{2}
\end{array}\right], \\
& a\left(1^{-}\right)=\left[\begin{array}{ccc}
G_{4} q^{2}+B_{6} \rho^{2} & \sqrt{2} B_{7} \rho^{2} & i \sqrt{2} B_{7}|q| \rho \\
\sqrt{2} B_{7} \rho^{2} & B_{8} \rho^{2} & i B_{8}|q| \rho \\
-i \sqrt{2} B_{7}|q| \rho & -i B_{8}|q| \rho & B_{8} q^{2}
\end{array}\right] \text {, } \\
& a\left(0^{+}\right)=\left[\begin{array}{cccc}
G_{5} q^{2}+B_{9} \rho^{2} & -i \sqrt{2} B_{9}|q| \rho & 0 & -i \sqrt{6} B_{11}|q| \rho \\
i \sqrt{2} B_{9}|q| \rho & B_{10} q^{2} & 0 & \sqrt{3} B_{12} q^{2} \\
0 & 0 & 0 & 0 \\
i \sqrt{6} B_{11}|q| \rho & \sqrt{3} B_{12} q^{2} & 0 & b_{0} q^{2}
\end{array}\right] \text {, } \\
& a\left(0^{-}\right)=G_{6} q^{2}+B_{13} \rho^{2},
\end{aligned}
$$


where

$$
\begin{array}{ll}
G_{1}=4 g_{1}+2 g_{2}+4 g_{3}+g_{4}+g_{5}, & B_{5}=4 a_{1}-2 a_{2}, \\
G_{2}=4 g_{1}+g_{2}, & B_{6}=2 a_{1}+a_{2}+2 a_{3}-g_{0}, \\
G_{3}=4 g_{1}-4 g_{3}+g_{4}-g_{5}, & B_{7}=a_{3}-g_{0}, \\
G_{4}=4 g_{1}+g_{2}+2 g_{4}, & B_{8}=2 a_{1}+a_{2}+a_{3}, \\
G_{5}=4 g_{1}+2 g_{2}+4 g_{3}+4 g_{4}+4 g_{5}, & B_{9}=2 a_{1}+a_{2}+3 a_{3}-2 g_{0}, \\
G_{6}=4 g_{1}-2 g_{2}, & B_{10}=4 a_{1}+2 a_{2}+6 a_{3}, \\
B_{1}=2 a_{1}+a_{2}+g_{0}, & B_{11}=b_{1}-2 g_{0}, \\
B_{2}=4 a_{1}+2 a_{2}, & B_{12}=2 b_{1}, \\
B_{3}=6 a_{1}-5 a_{2}-g_{0}, & B_{13}=8 a_{1}-8 a_{2}-2 g_{0}, \\
B_{4}=2 a_{1}-3 a_{2}-g_{0}, & B_{0}=b_{0} .
\end{array}
$$

We observe that if we did not redefine the fluctuation of the metric and worked with $\delta g$, the only effect on the coefficient matrices would be to multiply by $\rho / 2$ the second row and column of $a\left(2^{+}\right)$, the third row and column of $a\left(1^{-}\right)$and the second and third row and column of $a\left(0^{+}\right)$. This would change the determinants of these matrices by an overall power of $\rho^{2}$, which, as we shall see, does not affect the renormalization group equations.

There are a few checks that one can make on these matrices. First we observe that the matrices $a\left(1^{-}\right)$and $a\left(0^{+}\right)$are degenerate. The proportionality of the last two rows and columns of $a\left(1^{-}\right)$and the vanishing of the third row and column of $a\left(0^{+}\right)$are direct consequences of the diffeomorphism invariance.

If we take the only nonzero coefficients to be $g_{3}, g_{5}=-4 g_{3}, g_{6}=g_{3}$, corresponding to the action (2.3), the coefficient matrices are identically zero; this is because the corresponding action is a topological invariant (actually zero, since we expand around flat space). If we take the only nonzero coefficients to be $g_{0}, a_{1}=-\frac{1}{4} g_{0}, a_{2}=-\frac{1}{2} g_{0}, a_{3}=g_{0}$, $b_{0}$ and $b_{1}=2 g_{0}$, corresponding to the Lagrangian (2.5), the coefficient matrices reduce to

$$
\begin{aligned}
& a\left(2^{+}\right)=\left[\begin{array}{cc}
0 & 0 \\
0 & -2 g_{0} q^{2}
\end{array}\right], \\
& a\left(0^{+}\right)=\left[\begin{array}{cccc}
0 & 0 & 0 & 0 \\
0 & 4 g_{0} q^{2} & 0 & 4 \sqrt{3} g_{0} q^{2} \\
0 & 0 & 0 & 0 \\
0 & 4 \sqrt{3} g_{0} q^{2} & 0 & B_{0} q^{2}
\end{array}\right] .
\end{aligned}
$$

Note that for $g_{0}=b_{0} / 12$ the second and fourth rows and columns of $a\left(0^{+}\right)$are proportional, and the matrix has rank one. This is because in this case the action is Weyl invariant (see Appendix A). If we freeze $\rho=$ constant, the last row and column of $a\left(0^{+}\right)$can be suppressed and we are left with the familiar coefficient matrices of Einstein's theory [12].

The contribution of the potential to the inverse propagators is

$$
a\left(2^{+}\right)_{\mathrm{Pot}}=\left[\begin{array}{cc}
0 & 0 \\
0 & -\frac{2}{\rho^{2}} V
\end{array}\right],
$$




$$
\begin{aligned}
a\left(1^{-}\right)_{\text {Pot }} & =\left[\begin{array}{ccc}
0 & 0 & 0 \\
0 & 0 & 0 \\
0 & 0 & -\frac{2}{\rho^{2}} V
\end{array}\right], \\
a\left(0^{+}\right)_{\text {Pot }} & =\left[\begin{array}{cccc}
0 & 0 & 0 & 0 \\
0 & \frac{1}{\rho^{2}} V & \frac{\sqrt{3}}{\rho^{2}} V & \frac{\sqrt{3}}{\rho} \frac{d V}{d \rho} \\
0 & \frac{\sqrt{3}}{\rho^{2}} V & -\frac{1}{\rho^{2}} V & \frac{1}{\rho} \frac{d V}{d \rho} \\
0 & \frac{\sqrt{3}}{\rho} \frac{d V}{d \rho} & \frac{1}{\rho} \frac{d V}{d \rho} & \frac{d^{2} V}{d \rho^{2}}
\end{array}\right] .
\end{aligned}
$$

Note that these matrices do not have the degeneracies of $(3.11 d, e)$ or $(3.12 b)$. This is because flat space (with $\rho=$ constant) is a solution of the field equations only if $V=0$. Finally the contribution of the gauge fixing terms is

$$
\begin{aligned}
& a\left(1^{-}\right)_{\mathrm{GF}}=\left[\begin{array}{ccc}
0 & 0 & 0 \\
0 & 0 & 0 \\
0 & 0 & \frac{1}{2 \alpha} q^{2}
\end{array}\right], \\
& a\left(0^{+}\right)_{\mathrm{GF}}=\left[\begin{array}{cccc}
0 & 0 & 0 & 0 \\
0 & 0 & 0 & 0 \\
0 & 0 & \frac{1}{\alpha} q^{2} & 0 \\
0 & 0 & 0 & 0
\end{array}\right] .
\end{aligned}
$$

With these results, the usual one-loop effective action is equal to the sum over spin $J$ and parity $\mathcal{P}$ of the logarithms of the determinants of the matrices $a$. These are polynomials in $q^{2}, \rho^{2}, V$ and its derivatives of dimension up to eight. Taking into account the multiplicity of these contributions, the one-loop effective potential is

$$
V_{\text {eff }}(\rho)=\frac{1}{2} \sum_{J, \mathcal{P}}(2 J+1) \int \frac{d^{4} q}{(2 \pi)^{4}} \ln \left(\frac{\operatorname{det} a\left(J^{\mathcal{P}}\right)(\rho)}{\operatorname{det} a\left(J^{\mathcal{P}}\right)\left(\rho_{0}\right)}\right)
$$

We have normalized the effective action with the determinant of a free field, which is obtained by linearizing the action around a fixed constant field $\rho_{0}$. It is natural to choose $\rho_{0}$ as the minimun of $V_{\text {eff }}$ itself, in which case $V_{\text {eff }}\left(\rho_{0}\right)=0$.

Given the previous explicit form for the matrices $a\left(J^{\mathcal{P}}\right)$, one can now compute the expression for $V_{\text {eff }}(\rho)$, using standard renormalization techniques. As explained in the next section, we shall follow instead a different strategy: we shall derive the equation that describes the renormalization group flow of $V_{\text {eff }}$. This allows a more accurate discussion of the scale-dependence of the parameters that characterize the effective potential.

\section{Average effective potential}

To study the renormalization group flow of the effective potential, we shall use ideas

originally introduced by Wilson [13]. One begins from some action $S_{k_{1}}$ which is supposed to describe accurately the physics at some momentum scale $k_{1}$. Physics at a lower momentum 
scale $k_{2}$ is then described by an effective action $S_{k_{2}}$ which is obtained by functionally integrating $\exp \left(-S_{k_{1}}\right)$ over all fluctuations of the fields with momenta between $k_{1}$ and $k_{2}$. The procedure is then iterated: the effective action at scale $k_{3}<k_{2}$ is obtained by functionally integrating $\exp \left(-S_{k_{2}}\right)$ from $k_{2}$ to $k_{3}$, and so on. The flow of $S_{k}$ with $k$ is the renormalization group flow. Each step of the integration should not cover too large a range of momenta. In this way one can accurately compute the effective action at some low momentum scale $k$, starting from a high momentum scale $\Lambda$. Note that this is not the same as performing the functional integral from $\Lambda$ to $k$ in one step, because the action is updated at each step of the integration. In the following we will refer to this updating as "the renormalization group improvement".

Originally this idea was applied to spin fields on the lattice, but it was subsequently adapted to the continuum, where it was used to clarify and simplify the notion of renormalizability [14], and was also applied to gauge theories [15]. The particular implementation of this idea that we shall use here has been discussed in $[16,17]$. One can start from the usual definition of the effective action $S_{\text {eff }}$, defined as the Legendre transform of $W=-\ln Z$, where $Z$ is the partition function. The effective action has a perturbative expansion in Feynman diagrams, which correspond to integrals of certain functions of the momenta. By introducing some kind of infrared cutoff $k$ in the propagators one obtains a new effective action $S_{k}$, which depends on the scale $k$.

A way of implementing this idea in the path integral formalism is to add to the classical action $S$ a term $\Delta S_{k}$ that constrains the average of the field $\phi$ in spheres of radius $\approx k^{-1}$ centered around the point $x$ to be equal to a predetermined function $\bar{\phi}(x)$ (one can apply this discussion to the theory we are interested in by replacing the generic notation $\phi$ with $\Gamma, g$ and $\rho$ ). The average of a field $\phi$ around a point $x$ is defined by the convolution $f_{k} \phi(x)=\int d^{4} y \sqrt{\operatorname{det} g} f_{k}(x-y) \phi(y)$, or, when $g$ is flat, in Fourier space, by $f_{k} \phi(q)=f_{k}(q) \phi(q)$, where $f_{k}$ is the function

$$
f_{k}(q)=\exp \left(-a\left(\frac{q^{2}}{k^{2}}\right)^{b}\right),
$$

with $a$ and $b$ are constants. This function interpolates between a gaussian, for $b=1$, and a step function for $b \rightarrow \infty$.

In [10] we discussed this procedure in the context of a gauge theory of gravity where only the coefficients $g_{1}$ and $a_{1}$ were assumed to be nonzero. The specific choice of the term $\Delta S_{k}$ that we used there had the disadvantage that some of the propagators were not well defined. This was not important in [10] because these terms did not contribute to the quantities that we computed there. However, it could be a problem in more general calculations. For this reason we shall use here a simpler definition: we will assume that the term $\Delta S_{k}$ is defined in such a way that the only effect it has on the linearized action 
is to replace in the operators $\mathcal{O}$ the term $q^{2}$ with the function

$$
P_{k}(q)=\frac{q^{2}}{1-f_{k}(q)^{2}}
$$

(and $|q|$ by $\sqrt{P_{k}(q)}$ ). The function $P_{k}(q)$ approaches exponentially fast the function $q^{2}$ for $q^{2}>k^{2}$, but goes to a constant $(b=1)$ or diverges $(b>1)$ for $q^{2} \rightarrow 0$. Thus, replacing $q^{2}$ by $P_{k}$ in the propagators suppresses the modes with $q^{2}<k^{2}$ and therefore has an effect similar to putting an IR cutoff at momentum $k$. This definition of scale-dependent effective action $S_{k}$ is equivalent, at least at one-loop order, to the one given in [17].

It is quite clear that the considerations that were made in [17] for nonabelian gauge theories can be extended in a rather straightforward way to the case of gravity. There is one point, however, that requires some extra care: it is the definition of the absolute normalization of the potential. In the presence of gravity, the value of the potential at the minimum is interpreted as a cosmological constant. It affects the field equations, and therefore cannot be shifted arbitrarily. Furthermore, we see from (3.14) that it corresponds also to the mass of the graviton. In principle, the value of the potential at the minimum could depend on the scale, but one has to make sure that at least in the extreme IR limit this value be zero, to ensure that the graviton be massless.

We take the following definition of the scale dependent effective potential for $\rho$ :

$$
V_{k}(\rho)=\frac{1}{2} \sum_{J, \mathcal{P}}(2 J+1) \int \frac{d^{4} q}{(2 \pi)^{4}} \ln \left(\frac{\operatorname{det} a_{k}\left(J^{\mathcal{P}}\right)(\rho)}{\operatorname{det} a_{k}\left(J^{\mathcal{P}}\right)\left(\rho_{k}\right)}\right)
$$

where $a_{k}\left(J^{\mathcal{P}}\right)$ are obtained from the matrices $a\left(J^{\mathcal{P}}\right)$ given in $(3.11,14,15)$ by replacing $q^{2}$ with the function $P_{k}(q)$ and $\rho_{k}$ is defined to be the minimum of $V_{k}$. Note that with this definition $V_{k}\left(\rho_{k}\right)=0$ for all $k$, so that the cosmological constant is actually zero at all scales. In this connection see also [18].

Finally, we observe that since the determinants $\operatorname{det} a_{k}\left(J^{\mathcal{P}}\right)$ are functions of $\rho^{2}$, it is consistent to assume that $V_{k}$ is a function of $\rho^{2}$. It will be convenient to define $V^{\prime}=\frac{d V}{d\left(\rho^{2}\right)}$. Then in $(3.14 c)$ we can write $\frac{1}{\rho} \frac{d V}{d \rho}=2 V^{\prime}$ and $\frac{d^{2} V}{d \rho^{2}}=4 \rho^{2} V^{\prime \prime}+2 V^{\prime}$.

\section{Renormalization group flow}

The average effective potential obeys a renormalization group equation that is obtained from (4.3) by taking its derivative with respect to $k$. It has the form

$$
k \frac{d V_{k}}{d k}=F\left(V_{k}, V_{k}^{\prime}, V_{k}^{\prime \prime}\right)
$$

where the functional $F$ comes from the r.h.s. of (4.3):

$$
F\left(V, V^{\prime}, V^{\prime \prime}\right)=\frac{1}{2} \sum_{J, \mathcal{P}}(2 J+1) \int \frac{d^{4} q}{(2 \pi)^{4}} \frac{1}{\operatorname{det} a_{k}\left(J^{\mathcal{P}}\right)} k \frac{d}{d k}\left(\operatorname{det} a_{k}\left(J^{\mathcal{P}}\right)\right)
$$


with

$$
k \frac{d}{d k}\left(\operatorname{det} a_{k}\right)=\frac{\partial\left(\operatorname{det} a_{k}\right)}{\partial P_{k}} k \frac{\partial P_{k}}{\partial k} .
$$

After taking these derivatives, we substitute in $F$ the classical potential $V$ with the effective potential $V_{k}$ : this is the "renormalization group improvement". This substitution gives (5.1), a differential equation for the function $V_{k}(\rho)$. Notice that thanks to the behaviour of $P_{k}$ and its $k$-derivative in (5.3), the integral in (5.2) is actually dominated by a finite range of momenta and does not need an ultraviolet regularization.

Although derived in the context of a one loop approximation, this renormalization group improved equation has a validity that goes beyond one loop $[19,13,14]$. Clearly one cannot follow the evolution of the whole function $V_{k}$, so some other kind of approximation becomes necessary. In the following we shall study only the first few terms of the Taylor expansion of $V_{k}$. As explained in the introduction, we are interested in the spontaneously broken phase, with a nonzero VEV of $\rho$ at $k=0$. Thus, we parametrize $V_{k}$ by the position of its minimum, $\rho_{k}$, and the quartic coupling at the minimum $\lambda_{k}$ :

$$
V_{k}^{\prime}\left(\rho_{k}\right)=0, \quad \lambda_{k}=V_{k}^{\prime \prime}\left(\rho_{k}\right)
$$

In terms of these parameters the potential reads

$$
V_{k}(\rho)=\frac{1}{2} \lambda_{k}\left(\rho^{2}-\rho_{k}^{2}\right)^{2}
$$

which is the Taylor expansion of $V_{k}$ as function of $\rho^{2}$ around its minimum.

The equations governing the evolution in $k$ of $\rho_{k}^{2}$ and $\lambda_{k}$ can be obtained by differentiating the definitions (5.4). This leads to the following set of coupled partial differential equations:

$$
\begin{aligned}
& k \frac{\partial \rho_{k}^{2}}{\partial k}=\gamma(k) k^{2}, \\
& k \frac{\partial \lambda_{k}}{\partial k}=\beta(k),
\end{aligned}
$$

with

$$
\begin{aligned}
& \gamma(k)=-\frac{1}{\lambda k^{2}}\left(k \frac{\partial}{\partial k} V_{k}^{\prime}\right)\left(\rho_{k}\right)=\frac{1}{32 \pi^{2}} \int d x x \mathcal{R}_{\gamma}\left(P_{k}, \rho_{k}^{2}\right) k \frac{\partial P_{k}}{\partial k} \\
& \beta(k)=\left(k \frac{\partial}{\partial k} V_{k}^{\prime \prime}\right)\left(\rho_{k}\right)=\frac{1}{32 \pi^{2}} \int d x x \mathcal{R}_{\beta}\left(P_{k}, \rho_{k}^{2}\right) k \frac{\partial P_{k}}{\partial k}
\end{aligned}
$$

In the definition of $\beta(k)$ we are neglecting a term containing $V_{k}^{\prime \prime \prime}\left(\rho_{k}\right)$, which takes into account the $k$ dependence of the point of definition of $\lambda_{k}$ and is a signal of the presence of operators of higher dimension in the potential ("irrelevant" operators). Consistent with our approximation, we will neglect the effect of these terms. The functions $\beta$ and $\gamma$ have the general form shown in the r.h.s. of (5.7), where $x=q^{2}$ and $\mathcal{R}$ are rational functions of dimension $k^{-6}$. These functions can be computed explicitly from the representation 
(4.3) of $V_{k}$, by taking derivatives with respect to $\rho^{2}$ and $k$, and using (5.3). The general expressions are complicated and not particularly illuminating, so we will not give them here. It is not possible to find a solution of the system of p.d.e.'s (5.6-7) in closed form. However, analytic solutions can be obtained in some asymptotic limit.

Let us assume first that $\rho_{k}^{2}$ is small with respect to $k$. One would expect this to describe the behaviour of the theory in the regime when $k$ is large compared to the Planck mass. In simpler systems, this approximation indeed reproduces the results of perturbative calculations of beta functions at momenta much larger than the characteristic mass of the theory. Because of the factor $k \frac{\partial P_{k}}{\partial k}$, the integrals are dominated by the region $x \approx k^{2}$. In this region, $P_{k}$ is itself of order $k^{2}$, so that $\rho_{k}^{2}$ is small with respect to $P_{k}$. One can therefore expand the functions $\mathcal{R}$ in powers of $\rho_{k}^{2}$. In the function $\mathcal{R}_{\gamma}$ the dominant term is a constant (independent of $\rho_{k}$ ) but the function $\mathcal{R}_{\beta}$ has a pole for $\rho_{k} \rightarrow 0$, coming from the contribution of the spins $2^{+}, 1^{-}$and $0^{+}$. The beta functions reduce to the following:

$$
\gamma(k)=\gamma_{0}+O\left(\frac{\rho_{k}^{2}}{k^{2}}\right) \quad ; \quad \beta(k)=\beta_{-1} \frac{k^{2}}{\rho_{k}^{2}}+\beta_{0}+O\left(\frac{\rho_{k}^{2}}{k^{2}}\right),
$$

where

$$
\begin{aligned}
\gamma_{0}= & \frac{1}{32 \pi^{2}} \frac{1}{\lambda_{k}}\left[5 \frac{B_{1} B_{2}-2 B_{1}^{2}}{G_{1} B_{2}}+5 \frac{B_{1}}{G_{2}}+3 \frac{B_{3} B_{5}-2 B_{4}^{2}}{G_{3} B_{5}}+3 \frac{B_{6} B_{8}-2 B_{7}^{2}}{G_{4} B_{8}}\right. \\
& \left.+\frac{6 \lambda_{k}\left(B_{10}-2 B_{12}\right)}{B_{0} B_{10}-3 B_{12}^{2}}+\frac{B_{9}}{G_{5}}-\frac{2 B_{0} B_{9}^{2}-12 B_{9} B_{11} B_{12}+6 B_{10} B_{11}^{2}}{G_{5}\left(B_{0} B_{10}-3 B_{12}^{2}\right)}+\frac{B_{13}}{G_{6}}\right] I_{-2}, \\
\beta_{-1}= & \frac{1}{32 \pi^{2}}\left[\frac{10}{B_{2}}-\frac{B_{0}}{B_{0} B_{10}-3 B_{12}^{2}}+13 \alpha\right] \lambda_{k} I_{-2} \cdot \\
\beta_{0}= & \frac{1}{32 \pi^{2}}\left[10 \frac{\left(B_{1} B_{2}-2 B_{1}^{2}\right)^{2}+4 \lambda_{k} B_{1}^{2}}{G_{1}^{2} B_{2}^{2}}+10 \frac{B_{1}^{2}}{G_{2}^{2}}+6 \frac{\left(B_{3} B_{5}-2 B_{4}^{2}\right)^{2}}{G_{3}^{2} B_{5}^{2}}\right. \\
& +6 \frac{\left(B_{6} B_{8}-2 B_{7}^{2}\right)^{2}}{G_{4}^{2} B_{8}^{2}}+\frac{24\left(2 B_{0} B_{10}+3 B_{10}^{2}-12 B_{10} B_{12}+7 B_{12}^{2}\right)-16 \alpha B_{10}}{\left(B_{0} B_{10}-3 B_{12}^{2}\right)^{2}} \lambda_{k}^{2} \\
& +\frac{432\left(B_{10} B_{11}-B_{9} B_{12}\right)^{2}-4\left(B_{0} B_{9}-3 B_{11} B_{12}+12 B_{10} B_{11}-12 B_{9} B_{12}\right)^{2}}{G_{5}\left(B_{0} B_{10}-3 B_{12}^{2}\right)^{2}} \lambda_{k} \\
& \left.+\frac{2\left(2 B_{0} B_{9}^{2}-B_{0} B_{9} B_{10}+6 B_{10} B_{11}^{2}-12 B_{9} B_{11} B_{12}+3 B_{9} B_{12}^{2}\right)^{2}}{G_{5}^{2}\left(B_{0} B_{10}-3 B_{12}^{2}\right)^{2}}+2 \frac{B_{13}^{2}}{G_{6}^{2}}\right] I_{-3} .
\end{aligned}
$$

The positive dimensionless constants $I_{n}$ are defined by

$$
\begin{aligned}
& I_{-3}=\int d x x P_{k}^{-3} k \frac{\partial P_{k}}{\partial k}=1 \\
& I_{-2}=k^{-2} \int d x x P_{k}^{-2} k \frac{\partial P_{k}}{\partial k}=\frac{2}{(2 a)^{1 / b}} \Gamma(1+1 / b), \\
& I_{0}=k^{-6} \int d x x k \frac{\partial P_{k}}{\partial k}=\frac{2}{(2 a)^{3 / b}} \Gamma(1+3 / b) \zeta(3 / b) .
\end{aligned}
$$

Infrared convergence of $I_{0}$ requires that $b<3$. 
Note that in this approximation the dominant term in $\beta(k)$ is the one coming from the pole, unlike other known examples where the dominant term is the constant $\beta_{0}[16]$. This peculiarity can be traced to the presence of the undifferentiated potential $V$ in the inverse propagators, which is characteristic of gravity (cfr. (3.14)). The situation would be different if we allowed a nonzero cosmological constant.

Treating $\gamma_{0}, \beta_{-1}$ and $\beta_{0}$ as constants and neglecting integration constants one finds that $\rho_{k}^{2}=\frac{1}{2} \gamma_{0} k^{2}$ and $\lambda_{k}=2 \frac{\beta_{-1}}{\gamma_{0}} \ln k^{2}$. This is the behaviour that one would expect on dimensional grounds. In this calculation one neglects the running of the coupling constants. This is a reasonable approximation if one considers the behaviour of the theory over a range of momenta which is not too large. One could take into account the running of $\lambda_{k}$, for which the evolution equation is known, but the result would not be very significant: the other coupling constants are also expected to run logarithmically, and their (unknown) contribution could easily overwhelm the one coming from $\lambda_{k}$.

Assuming that all the couplings appearing in $\gamma_{0}$ run logarithmically, the true solution for $\rho_{k}^{2}$ would deviate from the one given above by a sublogarithmic correction. The validity of the approximation $\rho_{k}^{2} \ll k^{2}$ hinges on the sign of these corrections. If $\rho_{k}^{2}$ grows slower than $k^{2}$, the approximation could be justified. This was the case in the calculation we did in [10]. However, it may not be generally true.

Given that, in general, the validity of the approximation $\rho_{k}^{2} \ll k^{2}$ is questionable, it would be desirable to say something on the large- $k$ behaviour without making this assumption. One general conclusion that can be drawn with reasonable confidence is that $\rho_{k}^{2}$ will be proportional to $k^{2}$, up to (at most) logarithmic corrections. To see this consider again the general form (5.7) of the functions $\gamma(k)$ and $\beta(k)$. As mentioned before, the $x$-integration is cut off exponentially for $x>k^{2}$, and as a power for $x<k^{2}$, so if we are only interested in the dominant behaviour of the integral, we can replace $P_{k}$ by $k^{2}$. Assume further that $\rho_{k}^{2}=c k^{2}$ for some constant $c$. Then $\mathcal{R}\left(k^{2}, c k^{2}\right)$ is a constant that can be taken out of the $x$-integration and the functions $\gamma(k)$ and $\beta(k)$ become simply constants. The equation for $\rho_{k}^{2}$ becomes an algebraic equation that implicitly determines the constant $c$, so the assumption $\rho_{k}^{2}=c k^{2}$ is justified a posteriori.

Let us now consider the opposite limit: $k^{2} \ll \rho_{k}^{2}$. This is the limit $k \rightarrow 0$, when $\rho_{0} \neq 0$. In this case we retain in each sum the term with the highest power of $\rho_{k}^{2}$. The contributions of the different spins are not all of the same order in $k^{2} / \rho_{k}^{2}$. Keeping only the leading terms, the beta functions reduce to

$$
\gamma(k)=\gamma_{2} \frac{k^{4}}{\rho_{k}^{4}}+O\left(\frac{k^{6}}{\rho_{k}^{6}}\right) \quad ; \quad \beta(k)=\beta_{1} \frac{k^{2}}{\rho_{k}^{2}}+O\left(\frac{k^{4}}{\rho_{k}^{4}}\right) .
$$

where

$$
\begin{aligned}
\gamma_{2} & =\frac{1}{32 \pi^{2}} \frac{1}{\lambda_{k}}\left[5 \frac{G_{1} B_{2}}{B_{1} B_{2}-2 B_{1}^{2}}+5 \frac{G_{2}}{B_{1}}+3 \frac{G_{3} B_{5}}{B_{3} B_{5}-2 B_{4}^{2}}+3 \frac{G_{4} B_{8}}{B_{6} B_{8}-2 B_{7}^{2}}\right. \\
& -\frac{G_{5} B_{10}}{B_{9}}+\frac{6 G_{5} B_{9}\left(B_{10} B_{11}-B_{9} B_{12}\right)}{\left(B_{9} B_{10}-2 B_{9}^{2}\right)^{2}}+\frac{3 B_{0}\left(2 B_{9}-B_{10}-4 B_{11}+2 B_{12}\right)}{8 \lambda_{k}\left(2 B_{9}-B_{10}\right)} \\
& \left.+9 \frac{\left(2 B_{9}-B_{10}-4 B_{11}+2 B_{12}\right)\left(B_{9} B_{12}^{2}-4 B_{9} B_{11} B_{12}+2 B_{10} B_{11}^{2}\right)}{8 \lambda_{k} B_{9}\left(2 B_{9}-B_{10}\right)^{2}}+\frac{G_{6}}{B_{13}}\right] I_{0},
\end{aligned}
$$




$$
\beta_{1}=\frac{1}{32 \pi^{2}}\left[\frac{10}{B_{2}-2 B_{1}}-\frac{5}{2 B_{9}-B_{10}}+15 \alpha\right] I_{-2} \lambda_{k} .
$$

In this regime the running of $\lambda_{k}$ and $\rho_{k}^{2}$ is damped by powers of $k^{2} / \rho_{k}^{2}$ and stops for $k \rightarrow 0$. The solutions for small $k$ are

$$
\begin{aligned}
& \rho_{k}^{2}=\rho_{0}^{2}\left[1+\frac{\gamma_{2}}{6} \frac{k^{6}}{\rho_{0}^{6}}\right], \\
& \lambda_{k}=\lambda_{0}\left[1+\frac{\beta_{1}}{2} \frac{k^{2}}{\rho_{0}^{2}}\right] .
\end{aligned}
$$

For generic values of the parameters $g_{i}, a_{i}, b_{i}$, all modes except for the graviton are massive, with masses of the order of Planck's mass. One would expect that these modes can be neglected when describing the physics below the Planck scale. Thus, the running of the dilaton potential at low energies should be derivable entirely from the action (2.5). This can be easily checked using the coefficient matrices given in (3.12). The renormalization group equations become for small $k$

$$
\begin{aligned}
& k \frac{\partial \rho_{k}^{2}}{\partial k}=\frac{3}{32 \pi^{2}} \frac{12 g_{0}-B_{0}}{8 \lambda_{k}^{2}} I_{0} \frac{k^{6}}{\rho_{k}^{4}}, \\
& k \frac{\partial \lambda_{k}}{\partial k}=\frac{15}{32 \pi^{2}} \lambda_{k}\left(\alpha-\frac{1}{8 g_{0}}\right) I_{-2} \frac{k^{2}}{\rho_{k}^{2}} .
\end{aligned}
$$

These have again the solutions (5.16). Note that the coefficient $\gamma_{2}$ vanishes when $g_{0}=$ $b_{0} / 12$, in which case the action (2.5) is conformally invariant. (See Appendix A).

\section{Concluding remarks}

One attractive feature of the theory we have considered here is that one can easily describe the transition to Einstein's theory at sufficiently low energies. Indeed, for generic values of the coupling constants $g_{i}$ and $a_{i}$, all components of the connection $\Gamma_{\mu}{ }^{\nu}{ }_{\rho}$ are massive, with squared masses of the form $\rho_{0}^{2} B / G$, where $B$ and $G$ are appropriate combinations of the coupling constants $a_{i}$ and $g_{i}$. As mentioned in the introduction, this is due to the occurrence of a Higgs phenomenon. The dilaton becomes massive too, with a mass squared equal to $\lambda_{0} \rho_{0}^{2}$. At energies lower than the $\operatorname{VEV} \rho_{0}$, all these particles decouple, leaving the graviton as the only remnant. Its effective dynamics is given by the second term in (2.5). If we assume that the Einstein term of the low energy world comes entirely from this source, we see that $\rho_{0}$ has to be of the order of the Planck mass, $m_{P}$.

The results of the preceding section can now be summarized as follows: at scales $k$ much larger than $m_{P}$, the coupling constant $\lambda_{k}$ runs logarithmically and the VEV $\rho_{k}$ runs quadratically, as one would expect on dimensional grounds. These results are the reflection of the logarithmic and quadratic divergences that one would encounter if one tried to remove the ultraviolet cutoff from the theory. On the other hand for scales $k$ below the Planck mass the running is suppressed, and both $\lambda_{k}$ and $\rho_{k}$ tend to constants for $k \rightarrow 0$. 
When we said that there are massive particles in the theory we used in the mass formula the VEV of $\rho$ at the scale $k=0$. This is the naive procedure that one would follow, at tree level, with a classical potential. However, the running of the VEV of the dilaton affects the position of the pole of the propagators. Let us see this first in the case of the dilaton itself. From the parametrization (5.5) of the potential $V_{k}$, the running mass $m^{2}(k)=\left.\frac{d^{2} V_{k}}{d \rho^{2}}\right|_{\rho_{k}}$ is equal to $\lambda_{k} \rho_{k}^{2}$. The physical mass of the particle, i.e. the position of the pole of the propagator, is defined implicitly by the condition

$$
m_{\text {phys }}^{2}=\left.m^{2}(k)\right|_{k^{2}=m_{\text {phys }}^{2}} .
$$

This is equivalent to the statement, which was made in [10], that in a propagator of the form $\left(q^{2}-m^{2}(k)\right)^{-1}$ the pole is to be found by evaluating the running mass at $k=|q|$. Equation (6.1) is solved graphically, by finding the intersection of the plots of the l.h.s. $y=m_{\text {phys }}^{2}$ and the r.h.s. $y=\left.\lambda_{k} \rho_{k}^{2}\right|_{k^{2}=m_{\text {phys }}^{2}}$, as functions of the independent variable $m_{\text {phys }}^{2}$. The 1.h.s. is represented by the straight line at $45^{0}$. The r.h.s. is the plot of the running of $\rho_{k}$ that we have computed in the previous section, multiplied by a factor $\lambda_{0}$, up to logarithmic factors that we neglect for the moment. As we have seen, it starts flat at $\lambda_{0} \rho_{0}^{2}$ and grows linearly for large $m_{\text {phys }}^{2}$. If $\lambda_{0} \ll 1$, the intersection occurs in the region where the r.h.s. is constant, and therefore the mass squared is $\lambda_{0} \rho_{0}^{2} \ll m_{P}^{2}$. On the other hand if $\lambda_{0}$ is of the order or bigger than one, the intersection is shifted to higher energies. Exactly where it occurs depends crucially on the logarithmic corrections. If the leading logarithmic factors in the r.h.s. appear with a negative power, $m_{\text {phys }}^{2}$ is of the order $\exp \left(\lambda_{0}\right) \rho_{0}^{2}$. If they appear with a positive power, there may be no intersection at all. In this case, the dilaton would disappear completely from the spectrum. Note that an anomalous dimension of $\rho$ would give a power correction to the running of the mass, so it would be even more important than the logarithmic corrections in the previous considerations.

This discussion can be repeated for the connection $\Gamma_{\lambda}{ }^{\mu}{ }_{\nu}$, which potentially carries dangerous ghost or tachyon states. The running of $\rho_{k}$ could eliminate these states from the spectrum. That such a mechanism could exist was suggested in [20], but no concrete support for this idea had been given until now. In order to draw some definite conclusion one would have to compute the beta functions for the couplings $g_{i}$ and $a_{i}$ for large $k$, find the ultraviolet fixed point, if there is any, and evaluate (5.9) at that point.

The same discussion can be repeated also for any other matter field. In this theory the matter is supposed to be coupled to gravity in such a way that the masses of all particles are proportional to the VEV of the dilaton. For example, in the case of a scalar field $\phi$, the action would be

$$
S(\phi, g, \rho)=\frac{1}{2} \int d^{4} x \sqrt{\operatorname{det} g}\left[g^{\mu \nu} \partial_{\mu} \phi \partial_{\nu} \phi+h \rho^{2} \phi^{2}\right]
$$

where $h$ is a dimensionless coupling constant. The reason why the known particles have masses much smaller than Planck's mass would be the smallness of the coupling constant $h$. For such particles the intersection of the curves given by the l.h.s. and r.h.s. of (6.1) occurs in the region where the $\mathrm{VEV}$ of the dilaton is constant, so the poles are exactly where one 
would expect to find them. In GUT theories the expected masses are only a few orders of magnitude smaller than the Planck scale, so that the renormalization group corrections envisaged here may not be negligible. In the case of a scalar field, this is discussed in a separate publication [21].

Finally, we mention that the definition (4.3) of the scale dependent effective potential is not the only possibility. One could choose a different normalization of $V_{k}(\rho)$ such that its minimum (the cosmological constant) is actually $k$-dependent. These alternatives could be of relevance for example in cosmological problems.

\section{Acknowledgements}

R.P. wishes to thank G. Veneziano for the kind hospitality and support at the Theory Division of CERN, where part of this work was done. We are also grateful to I. Oda and M. Reuter for several discussions, and to F. Hehl for calling our attention on Ref. [23]. 


\section{Appendix A: Broken Weyl invariance}

Let us consider the behaviour of the action (2.2) under Weyl scalings

$$
g_{\mu \nu} \rightarrow \Omega^{2} g_{\mu \nu}, \quad \rho \rightarrow \Omega^{-1} \rho, \quad \Gamma_{\lambda}{ }^{\mu}{ }_{\nu} \rightarrow \Gamma_{\lambda}{ }^{\mu}{ }_{\nu}+\delta_{\nu}^{\mu} \Omega^{-1} \partial_{\lambda} \Omega,
$$

where $\Omega$ is a general function of the position. There is also another possible way in which a Weyl transformation could act on the connection: it is defined by requiring that $\Gamma$ transforms like the Christoffel symbols of $g$. This alternative transformation acts trivially on the torsion tensor, whereas (A.1) acts in a nontrivial way. We have chosen the transformation (A.1) because it can be generalized to a local $G L(4)$ transformation.

It is easy to see that the curvature tensor $R_{\mu \nu}{ }^{\rho} \sigma$ is inert under (A.1), so the curvature squared terms are invariant (as in Yang-Mills theory). The remaining terms can be made invariant by choosing

$$
b_{0}=6\left(2 a_{1}+a_{2}+3 a_{3}\right), \quad b_{1}=2 a_{1}+a_{2}+3 a_{3}, \quad V(\rho)=\frac{1}{2} \lambda \rho^{4},
$$

where $\lambda$ is an arbitrary constant. Note in particular that with the choices leading to (2.5) this gives $g_{0}=b_{0} / 12$.

It is sometimes convenient to write the action in a different form. Define the combinations

$$
\tilde{g}_{\mu \nu}=\rho^{2} g_{\mu \nu}, \quad \tilde{\Gamma}_{\lambda}{ }^{\mu}{ }_{\nu}=\Gamma_{\lambda}{ }^{\mu}{ }_{\nu}+\delta_{\nu}^{\mu} \rho^{-1} \partial_{\lambda} \rho,
$$

which by construction are inert under the transformation (A.1). We also define the cur-

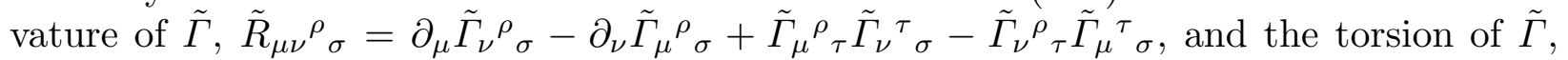
$\tilde{T}_{\lambda}{ }^{\mu}{ }_{\nu}=\tilde{\Gamma}_{\lambda}{ }^{\mu}{ }_{\nu}-\tilde{\Gamma}_{\nu}{ }^{\mu}{ }_{\lambda}$. The following relations hold:

$$
\begin{aligned}
\tilde{R}_{\mu \nu}{ }^{\rho}{ }_{\sigma} & =R_{\mu \nu}{ }^{\rho}{ }_{\sigma}, \\
\tilde{T}_{\lambda}{ }^{\mu}{ }_{\nu} & =T_{\lambda}{ }^{\mu}{ }_{\nu}+\delta_{\nu}^{\mu} \rho^{-1} \partial_{\lambda} \rho-\delta_{\lambda}^{\mu} \rho^{-1} \partial_{\nu} \rho, \\
\partial_{\lambda} \tilde{g}_{\mu \nu}-\tilde{\Gamma}_{\lambda}{ }^{\tau}{ }_{\mu} \tilde{g}_{\tau \nu}-\tilde{\Gamma}_{\lambda}{ }^{\tau}{ }_{\nu} \tilde{g}_{\mu \tau} & =\rho^{2}\left(\partial_{\lambda} g_{\mu \nu}-\Gamma_{\lambda}{ }^{\tau}{ }_{\mu} g_{\tau \nu}-\Gamma_{\lambda}{ }^{\tau}{ }_{\nu} g_{\mu \tau}\right) .
\end{aligned}
$$

Note from $(A .4 c)$ that if $\rho \neq 0$, metricity of one connection guarantees the metricity of the other.

With the relations $(A .2)$, the action $(2.3)$ can be written $S(g, \Gamma, \rho)=\tilde{S}(\tilde{g}, \tilde{\Gamma})$, where

$$
\begin{aligned}
& \tilde{S}(\tilde{g}, \tilde{\Gamma})=\int d^{4} x \sqrt{\operatorname{det} \tilde{g}}\left[\frac{1}{2} \lambda+g_{0} \tilde{R}+a_{1} \tilde{T}_{\mu \nu \rho} \tilde{T}^{\mu \nu \rho}+a_{2} \tilde{T}_{\mu \nu \rho} \tilde{T}^{\mu \rho \nu}+a_{3} \tilde{T}^{\rho} \tilde{T}_{\rho}\right. \\
& \left.+g_{1} \tilde{R}_{\mu \nu \rho \sigma} \tilde{R}^{\mu \nu \rho \sigma}+g_{2} \tilde{R}_{\mu \nu \rho \sigma} \tilde{R}^{\mu \rho \nu}+g_{3} \tilde{R}_{\mu \nu \rho \sigma} \tilde{R}^{\rho \sigma \mu \nu}+g_{4} \tilde{R}_{\mu \nu} \tilde{R}^{\mu \nu}+g_{5} \tilde{R}_{\mu \nu} \tilde{R}^{\nu \mu}+g_{6} \tilde{R}^{2}\right]
\end{aligned}
$$

where $\tilde{R}_{\mu \nu}=\tilde{R}_{\rho \mu}{ }^{\rho}{ }_{\nu}, \tilde{R}=\tilde{g}^{\mu \nu} \tilde{R}_{\mu \nu}$ and $\tilde{T}_{\nu}=\tilde{T}_{\nu}{ }^{\mu}{ }_{\mu}$. In this action indices are raised and lowered with $\tilde{g}$. This is the most general action quadratic in $\tilde{T}$ and $\tilde{R}$, and it is invariant under the transformations (A.1) in a trivial way, since all quantities entering in this action are inert under those transformations. It is obtained from (2.2) by choosing the conformal gauge in which $\rho$ is constant, and rescaling the fields by factors $\rho$. Conversely, (2.2) with 
the relations $(A .2)$ is obtained from $(A .5)$ by using the definitions $(A .3)$. Note that the field $\rho$, which we call the dilaton in this paper, may be called the conformal factor of the metric $\tilde{g}$ if one started from the action $(A .5)$. In the literature on conformal gravity the two actions (2.3)-(A.2) and $(A .5)$ are said to be written in the Jordan and Einstein conformal frames, respectively. They are completely equivalent at the classical level.

Let us now consider the quantization of the theory in the Weyl-invariant case. We note first of all that when $(A .2)$ holds, $B_{11}=B_{9}$ and $B_{12}=B_{10}$. Thus at the linearized level the new gauge invariance manifests itself in the proportionality of the second and fourth rows and columns in $a\left(0^{+}\right)$. Therefore, one has to fix the gauge also for Weyl transformations. Assuming that this has been done, it is easy to see that there exists a quantization procedure that preserves Weyl invariance. One has to use the form $(A .5)$ for the action, and define the functional measure by means of the metric $\tilde{g}$. In this case the effective action can be written again as a functional of $\tilde{g}$ and $\tilde{\Gamma}$ alone, and therefore is automatically invariant under $(A .1)[22]$.

One is naturally inclined to preserve as much as possible the classical symmetries in the quantization process, so this choice of measure may seem to be the only sensible one. However, this is not the case. Other choices are possible and, from a certain point of view, may even be more natural: if we interpret the metric $g$ as the one defining the geometry of spacetime, then it is natural to use $g$ rather than $\tilde{g}$ in the definition of the functional measure, and this leads to a quantum theory in which Weyl invariance is broken.

In a concrete calculation, the definition of the measure reflects itself in the definition of the cutoff. We have shown in $[10,11]$, that if one starts from a Weyl invariant theory and defines the UV momentum cutoff as $g^{\mu \nu} q_{\mu} q_{\nu}<\Lambda^{2}$ (rather than $\tilde{g}^{\mu \nu} q_{\mu} q_{\nu}<\Lambda^{2}$ ), then the effective action $S_{\text {eff }}(g, \Gamma, \rho)$ will not have Weyl invariance anymore.

In this paper we have only discussed the renormalization group flow of the effective action, which does not necessitate the explicit introduction of an UV cutoff. Still, Weyl invariance cannot be maintained. This can be seen as follows. Suppose we study the small fluctuations of the gravitational field around flat space at some energy scale $k_{1}$, and suppose that these are well described by the Weyl invariant action (2.3)-(A.2). Now suppose we want to know the effective action at some lower energy scale $k_{2}$. As discussed in section 4, this is given by a functional integral over all fluctuations of the fields which lie in the momentum shell between $k_{1}$ and $k_{2}$. But how is this momentum shell defined? Since we are postulating that the geometry of spacetime is given by the dimensionless metric $g$, the shell is defined by $k_{2}<g^{\mu \nu} q_{\mu} q_{\nu}<k_{1}$ (for simplicity we are assuming here a sharp cutoff, but this is by no means essential). One sees that the definition of the shell introduces in the definition of the effective action $S_{k_{2}}$ a dependence on $g$ which is not compensated by a dependence on $\rho$. Unavoidably, $S_{k}$ will not be a function of the combinations (2.4) alone. It will be a genuine functional of $g, \Gamma$ and $\rho$, and will not be invariant under infinitesimal Weyl transformations.

To be more specific, suppose that we want to compute only the scale-dependent effective potential $V_{k}(\rho)$, which is given by $S_{k}=\int d^{4} x \sqrt{\operatorname{det} g} V_{k}$, for a field of the form (3.1). As we have mentioned above, Weyl invariance requires the potential to be purely quartic. If we assume that $V_{k_{1}}$ is purely quartic, then unavoidably $V_{k_{2}}$ will not be anymore, because the integration procedure breaks scale invariance. It is instructive to see this in detail in an 
explicit calculation. Consider a single wave operator of the form $\mathcal{O}=q^{2}+c \rho^{2}$ (in principle (3.3) can be written as a sum of such terms), and suppose the momentum shell is defined by sharp UV and IR cutoffs. Then we have

$$
\begin{aligned}
\Delta V= & V_{k_{1}}-V_{k_{2}}=-\frac{1}{2} \int_{q^{2}=k_{2}^{2}}^{k_{1}^{2}} \frac{d^{4} q}{(2 \pi)^{4}} \ln \left(\frac{q^{2}+c \rho^{2}}{q^{2}+c \rho_{k_{1}}^{2}}\right) \\
= & \frac{1}{64 \pi^{2}}\left[k_{2}^{4} \ln \left(\frac{k_{2}^{2}+c \rho^{2}}{k_{2}^{2}+c \rho_{k_{1}}^{2}}\right)-k_{1}^{4} \ln \left(\frac{k_{1}^{2}+c \rho^{2}}{k_{1}^{2}+c \rho_{k_{1}}^{2}}\right)\right. \\
& \left.+c^{2} \rho^{4} \ln \left(\frac{k_{1}^{2}+c \rho^{2}}{k_{2}^{2}+c \rho^{2}}\right)-c^{2} \rho_{k_{1}}^{4} \ln \left(\frac{k_{1}^{2}+c \rho_{k_{1}}^{2}}{k_{2}^{2}+c \rho_{k_{1}}^{2}}\right)+c\left(\rho^{2}-\rho_{k_{1}}^{2}\right)\left(k_{2}^{2}-k_{1}^{2}\right)\right],
\end{aligned}
$$

which is obviously no longer purely quartic. It is still a function of $\rho^{2}$ only.

Let us approximate the potential $V_{k}$ by a quartic polynomial of the form

$$
V_{k}(\rho)=\frac{1}{2} m_{k}^{2} \rho^{2}+\lambda_{k} \rho^{4} .
$$

The constants $m_{k}^{2}$ and $\lambda_{k}$ are to be thought of as the coefficients of the Taylor expansion of $V_{k}$ around the origin:

$$
m_{k}^{2}=\left.\frac{d^{2} V_{k}}{d \rho^{2}}\right|_{0}, \quad \lambda_{k}=\left.\frac{1}{24} \frac{d^{4} V_{k}}{d \rho^{4}}\right|_{0} .
$$

Then we have

$$
\begin{gathered}
\Delta m_{k}^{2}=m_{k_{1}}^{2}-m_{k_{2}}^{2}=\frac{c}{32 \pi^{2}}\left(k_{2}^{2}-k_{1}^{2}\right), \\
\Delta \lambda_{k}=\lambda_{k_{1}}-\lambda_{k_{2}}=\frac{c^{2}}{64 \pi^{2}} \ln \frac{k_{1}^{2}}{k_{2}^{2}} .
\end{gathered}
$$

The most important conclusion of this discussion is that the potential cannot be consistently assumed to be purely quartic at all scales: if $m^{2}$ is zero at some scale, it will be nonzero as soon as one begins to integrate. Weyl invariance is broken and a mass term is generated. There follows that if we want to study the renormalization group flow of the effective potential taking into account what we called the "renormalization group improvement", we have to assume from the outset that the potential is not purely quartic. Of course, if we were to study the whole effective action rather than just the effective potential we would find many more terms that are not present in the starting action. For example, instead of the factors $\rho^{2}$ in front of the torsion terms there will now be general functions of $\rho$. In principle, this will also have an effect on the running of the potential, but we neglect this effect. 


\section{Appendix B: Lorentz decomposition}

In the main text we have taken as independent coupling constants the coefficients of all possible contractions of two curvature or torsion tensors. However, these contractions do not carry any special geometrical significance. One may expect that the final results would look simpler if they were expressed in terms of another set of parameters, which are related to geometrically significant quantities. Let us consider the irreducible parts of the torsion and curvature tensors with respect to the Lorentz group [23]:

$$
T_{\lambda \mu \nu}=\sum_{i=1}^{3} T_{\lambda \mu \nu}^{(i)} \quad, \quad R_{\mu \nu \rho \sigma}=\sum_{i=1}^{6} R_{\mu \nu \rho \sigma}^{(i)}
$$

where

$$
\begin{aligned}
T_{\lambda \mu \nu}^{(1)} & =\frac{1}{3}\left(2 T_{\lambda \mu \nu}-T_{\nu \lambda \mu}+T_{\nu \mu \lambda}\right)+\frac{1}{3}\left(g_{\lambda \mu} T_{\nu}-g_{\nu \mu} T_{\lambda}\right) \\
T_{\lambda \mu \nu}^{(2)} & =-\frac{1}{3}\left(g_{\lambda \mu} T_{\nu}-g_{\nu \mu} T_{\lambda}\right), \\
T_{\lambda \mu \nu}^{(3)} & =\frac{1}{3}\left(T_{\lambda \mu \nu}+T_{\nu \lambda \mu}+T_{\mu \nu \lambda}\right),
\end{aligned}
$$

and

$$
\begin{aligned}
& R_{\mu \nu \rho \sigma}^{(1)}=\frac{1}{6}\left(2 R_{\mu \nu \rho \sigma}+2 R_{\rho \sigma \mu \nu}-R_{\mu \rho \sigma \nu}+R_{\mu \sigma \rho \nu}+R_{\nu \rho \sigma \mu}-R_{\sigma \nu \rho \mu}\right) \\
& -\frac{1}{2}\left(g_{\mu \rho} R_{\nu \sigma}^{(\mathrm{ST})}-g_{\nu \rho} R_{\mu \sigma}^{(\mathrm{ST})}-g_{\mu \sigma} R_{\nu \rho}^{(\mathrm{ST})}+g_{\nu \sigma} R_{\mu \rho}^{(\mathrm{ST})}\right) \\
& -\frac{1}{12}\left(g_{\mu \rho} g_{\nu \sigma}-g_{\mu \sigma} g_{\nu \rho}\right) R \\
& R_{\mu \nu \rho \sigma}^{(2)}=\frac{1}{2}\left(R_{\mu \nu \rho \sigma}-R_{\rho \sigma \mu \nu}\right)-\frac{1}{2}\left(g_{\mu \rho} R_{\nu \sigma}^{(\mathrm{A})}-g_{\nu \rho} R_{\mu \sigma}^{(\mathrm{A})}-g_{\mu \sigma} R_{\nu \rho}^{(\mathrm{A})}\right. \\
& \left.+g_{\nu \sigma} R_{\mu \rho}^{(\mathrm{A})}\right) \\
& R_{\mu \nu \rho \sigma}^{(3)}=\frac{1}{6}\left(R_{\mu \nu \rho \sigma}+R_{\rho \sigma \mu \nu}+R_{\mu \sigma \nu \rho}+R_{\nu \rho \mu \sigma}+R_{\mu \rho \sigma \nu}+R_{\sigma \nu \mu \rho}\right), \\
& R_{\mu \nu \rho \sigma}^{(4)}=\frac{1}{2}\left(g_{\mu \rho} R_{\nu \sigma}^{(\mathrm{ST})}-g_{\nu \rho} F_{\mu \sigma}^{(\mathrm{ST})}-g_{\mu \sigma} R_{\nu \rho}^{(\mathrm{ST})}+g_{\nu \sigma} R_{\mu \rho}^{(\mathrm{ST})}\right) \\
& -\frac{1}{12}\left(g_{\mu \rho} g_{\nu \sigma}-g_{\mu \sigma} g_{\nu \rho}\right) R \\
& R_{\mu \nu \rho \sigma}^{(5)}=\frac{1}{2}\left(g_{\mu \rho} R_{\nu \sigma}^{(\mathrm{A})}-g_{\nu \rho} R_{\mu \sigma}^{(\mathrm{A})}-g_{\mu \sigma} R_{\nu \rho}^{(\mathrm{A})}+g_{\nu \sigma} R_{\mu \rho}^{(\mathrm{A})}\right), \\
& R_{\mu \nu \rho \sigma}^{(6)}=\frac{1}{12}\left(g_{\mu \rho} g_{\nu \sigma}-g_{\mu \sigma} g_{\nu \rho}\right) F
\end{aligned}
$$

where $R_{\mu \nu}^{(\mathrm{ST})}=\frac{1}{2}\left(R_{\mu \nu}+R_{\nu \mu}\right)-\frac{1}{4} g_{\mu \nu} F$ and $R_{\mu \nu}^{(\mathrm{A})}=\frac{1}{2}\left(R_{\mu \nu}-R_{\nu \mu}\right)$ are the symmetric traceless and antisymmetric parts of the Ricci tensor. These decompositions are orthogonal 
with respect to the inner products $(A, B)=\int d^{4} x \sqrt{\operatorname{det} g} g^{\mu \rho} g^{\nu \sigma} g^{\lambda \tau} \cdots A_{\mu \nu \lambda \ldots} B_{\rho \sigma \tau \ldots}$. The quantity (2.6) can then be rewritten in the form

$$
\frac{1}{2} \sum_{i=1}^{6} \tilde{g}_{i} R_{\mu \nu \rho \sigma}^{(i)} R^{(i) \mu \nu \rho \sigma}+\frac{1}{2} \rho^{2} \sum_{i=1}^{3} \tilde{a}_{i} T_{\lambda \mu \nu}^{(i)} T^{(i) \lambda \mu \nu} .
$$

where $\tilde{a}_{i}$ and $\tilde{g}_{i}$ is a new set of coupling constants, related to the coupling constants $a_{i}, g_{i}$ appearing in the action (2.2) by the linear transformation

$$
\left[\begin{array}{l}
\tilde{g}_{1} \\
\tilde{g}_{2} \\
\tilde{g}_{3} \\
\tilde{g}_{4} \\
\tilde{g}_{5} \\
\tilde{g}_{6}
\end{array}\right]=\left[\begin{array}{cccccc}
1 & 1 & 1 / 2 & 0 & 0 & 0 \\
1 & -1 & 0 & 0 & 0 & 0 \\
1 & 1 & -1 & 0 & 0 & 0 \\
1 & 2 & 1 / 2 & 1 / 2 & 1 / 2 & 0 \\
1 & -1 & 0 & 1 / 2 & -1 / 2 & 0 \\
1 & 1 & 1 / 2 & 3 / 2 & 3 / 2 & 6
\end{array}\right]\left[\begin{array}{c}
g_{1} \\
g_{2} \\
g_{3} \\
g_{4} \\
g_{5} \\
g_{6}
\end{array}\right] \quad, \quad\left[\begin{array}{c}
\tilde{a}_{1} \\
\tilde{a}_{2} \\
\tilde{a}_{3}
\end{array}\right]=\left[\begin{array}{ccc}
2 & 1 & 0 \\
2 & 1 & 3 \\
2 & -2 & 0
\end{array}\right]\left[\begin{array}{l}
a_{1} \\
a_{2} \\
a_{3}
\end{array}\right]
$$

Most formulae are more compactly written in terms of the new coupling constants. This is evident from the following table, which gives the relation between the coefficients $G_{i}$ and $B_{i}$ appearing in (3.11) and the parameters appearing in the Lagrangian:

$$
\begin{array}{ll}
G_{1}=4 g_{1}+2 g_{2}+4 g_{3}+g_{4}+g_{5} & =(1 / 2) \tilde{g}_{1}+\tilde{g}_{4}, \\
G_{2}=4 g_{1}+g_{2} & =\tilde{g}_{1}+\tilde{g}_{2}, \\
G_{3}=4 g_{1}-4 g_{3}+g_{4}-g_{5} & =\tilde{g}_{2}+\tilde{g}_{5}, \\
G_{4}=4 g_{1}+g_{2}+2 g_{4} & =\tilde{g}_{4}+\tilde{g}_{5}, \\
G_{5}=4 g_{1}+2 g_{2}+4 g_{3}+4 g_{4}+4 g_{5}+12 g_{6} & =2 \tilde{g}_{6}+8 \tilde{g}_{4}-5 \tilde{g}_{1}, \\
G_{6}=4 g_{1}-2 g_{2} & =(1 / 3) \tilde{g}_{1}+\tilde{g}_{2}, \\
B_{1}=2 a_{1}+a_{2}+g_{0} & =\tilde{a}_{1}+g_{0}, \\
B_{2}=4 a_{1}+2 a_{2} & =2 \tilde{a}_{1}, \\
B_{3}=6 a_{1}-5 a_{2}-g_{0} & =(1 / 3)\left(\tilde{a}_{1}+8 \tilde{a}_{3}\right)-g_{0}, \\
B_{4}=2 a_{1}-3 a_{2}-g_{0} & =(1 / 3)\left(4 \tilde{a}_{3}-\tilde{a}_{1}\right)-g_{0}, \\
B_{5}=4 a_{1}-2 a_{2} & =(1 / 3)\left(2 \tilde{a}_{1}+4 \tilde{a}_{3}\right), \\
B_{6}=2 a_{1}+a_{2}+2 a_{3}-g_{0} & =(1 / 3)\left(\tilde{a}_{1}+2 \tilde{a}_{2}\right)-g_{0}, \\
B_{7}=a_{3}-g_{0} & =(1 / 3)\left(\tilde{a}_{2}-\tilde{a}_{1}\right)-g_{0}, \\
B_{8}=2 a_{1}+a_{2}+a_{3} & =(1 / 3)\left(2 \tilde{a}_{1}+\tilde{a}_{2}\right), \\
B_{9}=2 a_{1}+a_{2}+3 a_{3}-2 g_{0} & =\tilde{a}_{2}-2 g_{0}, \\
B_{10}=4 a_{1}+2 a_{2}+6 a_{3} & =2 \tilde{a}_{2}, \\
B_{11}=b_{1}-2 g_{0} & =b_{1}-2 g_{0}, \\
B_{12}=2 b_{1} & =2 b_{1}, \\
B_{13}=8 a_{1}-8 a_{2}-2 g_{0} & =4 \tilde{a}_{3}-2 g_{0} .
\end{array}
$$




\section{Appendix C: Spin-projector operators}

For completeness, we list in this appendix the explicit expressions of the spin-projector operators $P_{i j}^{A B}\left(J^{\mathcal{P}}\right)$ that have been used to rewrite the action (3.3) into the form (3.10). There are in fact some differences with respect to those in [10], due to the different spinparity content of the fields appearing here.

For fixed spin $J$ and parity $\mathcal{P}$, these operators are labelled by the indices $A, B$ that identify the fields $\omega, \varphi$ and $\sigma$, and by $i, j$ that identify isomorphic Lorentz representations occurring more than once. For example for spin-parity $2^{+}, i=1,2$; for $1^{-}, i=1,2,3$ etc. The operators $P_{i i}^{A A}\left(J^{\mathcal{P}}\right)$ project out of a field a given irreducible representation, while the intertwiners $P_{i j}^{A B}\left(J^{\mathcal{P}}\right)$ (with $i \neq j$ ) give isomorphisms between the different representations occurring more than once. (Note that the indices $A, B$ in these projectors are redundant since $i, j$ already label the representations. It is nevertheless convenient to keep them in order to remember by what field a certain representation is carried, e.g. for $J^{\mathcal{P}}=1^{-}$the representations $i=1$ and $i=2$ are carried by $\omega$ and $i=3$ is carried by $\varphi$ ). The operators $P_{i j}^{A B}\left(J^{\mathcal{P}}\right)$ are orthonormal and complete:

$$
\begin{aligned}
& P_{i j}^{A B}\left(J^{\mathcal{P}}\right) \cdot P_{k l}^{C D}\left(I^{\mathcal{Q}}\right)=\delta_{I J} \delta_{\mathcal{P} \mathcal{Q}} \delta_{j k} \delta_{B C} P_{i l}^{A D}\left(J^{\mathcal{P}}\right), \\
& \sum_{J, \mathcal{P}, A, i} P_{i i}^{A A}\left(J^{\mathcal{P}}\right)=\mathbf{1}
\end{aligned}
$$

It is useful to introduce the following notations:

$$
\hat{q}^{\mu}=q^{\mu} / \sqrt{q^{2}}, \quad L_{\mu}^{\nu}=\hat{q}_{\mu} \hat{q}^{\nu}, \quad T_{\mu}^{\nu}=\delta_{\mu}^{\nu}-L_{\mu}^{\nu},
$$

obeying the relations:

$$
L_{\nu}^{\mu} T_{\mu}^{\rho}=0, \quad T_{\mu}^{\nu} T_{\nu}^{\rho}=L_{\mu}^{\rho}, \quad L_{\mu}^{\nu} L_{\nu}^{\rho}=L_{\mu}^{\rho} .
$$

In terms of $\hat{q}^{\mu}, L_{\mu}^{\nu}$, and $T_{\mu}^{\nu}$, one finds:

$$
\begin{aligned}
& {\left[P\left(2^{+}\right)\right]=\left[\begin{array}{cc}
{\left[P_{11}^{\omega \omega}\left(2^{+}\right)\right]_{\tau \rho \sigma}{ }^{\alpha \beta \gamma}} & {\left[P_{12}^{\omega \varphi}\left(2^{+}\right)\right]_{\tau \rho \sigma}{ }^{\alpha \beta}} \\
{\left[P_{21}^{\varphi \omega}\left(2^{+}\right)\right]_{\rho \sigma}{ }^{\alpha \beta \gamma}} & {\left[P_{22}^{\varphi \varphi}\left(2^{+}\right)\right]_{\rho \sigma}{ }^{\alpha \beta}}
\end{array}\right]} \\
& {\left[P_{11}^{\omega \omega}\left(2^{+}\right)\right]_{\tau \rho \sigma}{ }^{\alpha \beta \gamma}=T_{\tau}^{\alpha} T_{[\rho}^{[\beta} L_{\sigma]}^{\gamma]}+T_{\tau}^{[\gamma} L_{[\rho}^{\beta]} T_{\sigma]}^{\alpha}-\frac{2}{3} T_{\tau[\rho} L_{\sigma]}^{[\gamma} T^{\beta] \alpha},} \\
& {\left[P_{12}^{\omega \varphi}\left(2^{+}\right)\right]_{\tau \rho \sigma}{ }^{\alpha \beta}=\sqrt{2} T_{\tau}^{(\alpha} T_{[\rho}^{\beta)} \hat{q}_{\sigma]}-\frac{\sqrt{2}}{3} T^{\alpha \beta} T_{\tau[\rho} \hat{q}_{\sigma]},} \\
& {\left[P_{21}^{\varphi \omega}\left(2^{+}\right)\right]_{\rho \sigma}{ }^{\alpha \beta \gamma}=\sqrt{2} T_{(\rho}^{\alpha} T_{\sigma)}^{[\beta} \hat{q}^{\gamma]}-\frac{\sqrt{2}}{3} T_{\rho \sigma} T^{\alpha[\beta} \hat{q}^{\gamma]},} \\
& {\left[P_{22}^{\varphi \varphi}\left(2^{+}\right)\right]_{\rho \sigma}{ }^{\alpha \beta}=T_{(\rho}^{(\alpha} T_{\sigma)}^{\beta)}-\frac{1}{3} T_{\rho \sigma} T^{\alpha \beta},} \\
& {\left[P^{\omega \omega}\left(2^{-}\right)\right]_{\tau \rho \sigma}{ }^{\alpha \beta \gamma}=\frac{2}{3} T_{\tau}^{\alpha} T_{[\rho}^{[\beta} T_{\sigma]}^{\gamma]}+\frac{2}{3} T_{\tau}^{[\gamma} T_{[\rho}^{\beta]} T_{\sigma]}^{\alpha}-T_{\tau[\rho} T_{\sigma]}^{[\gamma} T^{\beta] \alpha},}
\end{aligned}
$$




$$
\begin{aligned}
& {\left[P\left(1^{+}\right)\right]=\left[\begin{array}{lll}
{\left[P_{11}^{\omega \omega}\left(1^{+}\right)\right]_{\tau \rho \sigma}{ }^{\alpha \beta \gamma}} & {\left[P_{12}^{\omega \omega}\left(1^{+}\right)\right]_{\tau \rho \sigma}{ }^{\alpha \beta \gamma}} \\
{\left[P_{21}^{\omega \omega}\left(1^{+}\right)\right]_{\tau \rho \sigma}{ }^{\alpha \beta \gamma}} & {\left[P_{22}^{\omega \omega}\left(1^{+}\right)\right]_{\tau \rho \sigma}{ }^{\alpha \beta \gamma}}
\end{array}\right]} \\
& {\left[P_{11}^{\omega \omega}\left(1^{+}\right)\right]_{\tau \rho \sigma}{ }^{\alpha \beta \gamma}=T_{\tau}^{\alpha} T_{[\rho}^{[\beta} L_{\sigma]}^{\gamma]}-T_{\tau}^{[\gamma} L_{[\rho}^{\beta]} T_{\sigma]}^{\alpha},} \\
& {\left[P_{12}^{\omega \omega}\left(1^{+}\right)\right]_{\tau \rho \sigma}{ }^{\alpha \beta \gamma}=\sqrt{2} T_{\tau}^{[\gamma} T_{[\rho}^{\beta]} L_{\sigma]}^{\alpha},} \\
& {\left[P_{21}^{\omega \omega}\left(1^{+}\right)\right]_{\tau \rho \sigma}{ }^{\alpha \beta \gamma}=\sqrt{2} L_{\tau}^{[\gamma} T_{[\rho}^{\beta]} T_{\sigma]}^{\alpha},} \\
& {\left[P_{22}^{\omega \omega}\left(1^{+}\right)\right]_{\tau \rho \sigma}{ }^{\alpha \beta \gamma}=L_{\tau}^{\alpha} T_{[\rho}^{[\beta} T_{\sigma]}^{\gamma]},} \\
& {\left[P\left(1^{-}\right)\right]=\left[\begin{array}{cccc}
{\left[P_{11}^{\omega \omega}\left(1^{-}\right)\right]_{\tau \rho \sigma}{ }^{\alpha \beta \gamma}} & {\left[P_{12}^{\omega \omega}\left(1^{-}\right)\right]_{\tau \rho \sigma}{ }^{\alpha \beta \gamma}} & {\left[P_{13}^{\omega \varphi}\left(1^{-}\right)\right]_{\tau \rho \sigma}{ }^{\alpha \beta}} \\
{\left[P_{21}^{\omega \omega}\left(1^{-}\right)\right]_{\tau \rho \sigma}{ }_{\alpha \beta \gamma}} & {\left[P_{22}^{\omega \omega}\left(1^{-}\right)\right]_{\tau \rho \sigma}{ }_{\alpha \beta \gamma}} & {\left[P_{23}^{\omega \varphi}\left(1^{-}\right)\right]_{\tau \rho \sigma}{ }^{\alpha \beta}} \\
{\left[P_{31}^{\varphi \omega}\left(1^{-}\right)\right]_{\rho \sigma}{ }^{\alpha \beta \gamma}} & {\left[P_{32}^{\varphi \omega}\left(1^{-}\right)\right]_{\rho \sigma}{ }^{\alpha \beta \gamma}} & {\left[P_{33}^{\varphi \varphi}\left(1^{-}\right)\right]_{\rho \sigma}{ }^{\alpha \beta}}
\end{array}\right],} \\
& {\left[P_{11}^{\omega \omega}\left(1^{-}\right)\right]_{\tau \rho \sigma}{ }^{\alpha \beta \gamma}=T_{\tau[\rho} T_{\sigma]}^{[\gamma} T^{\beta] \alpha},} \\
& {\left[P_{12}^{\omega \omega}\left(1^{-}\right)\right]_{\tau \rho \sigma}{ }^{\alpha \beta \gamma}=\sqrt{2} L^{\alpha[\beta} T_{\sigma]}^{[\gamma} T_{\rho] \tau},} \\
& {\left[P_{13}^{\omega \varphi}\left(1^{-}\right)\right]_{\tau \rho \sigma}^{\alpha \beta}=\sqrt{2} T_{\tau[\rho} T_{\sigma]}^{(\alpha} \hat{q}^{\beta)} \text {, }} \\
& {\left[P_{21}^{\omega \omega}\left(1^{-}\right)\right]_{\tau \rho \sigma}{ }^{\alpha \beta \gamma}=\sqrt{2} L_{\tau[\rho} T_{\sigma]}^{[\gamma} T^{\beta] \alpha} \text {, }} \\
& {\left[P_{22}^{\omega \omega}\left(1^{-}\right)\right]_{\tau \rho \sigma}{ }^{\alpha \beta \gamma}=2 L_{\tau}^{\alpha} L_{[\rho}^{[\beta} T_{\sigma]}^{\gamma]},} \\
& {\left[P_{23}^{\omega \varphi}\left(1^{-}\right)\right]_{\tau \rho \sigma}^{\alpha \beta}=2 \hat{q}_{\tau} L_{[\rho}^{(\alpha} T_{\sigma]}^{\beta)} \text {, }} \\
& {\left[P_{31}^{\varphi \omega}\left(1^{-}\right)\right]_{\rho \sigma}{ }^{\alpha \beta \gamma}=\sqrt{2} T^{\alpha[\beta} T_{(\rho}^{\gamma]} \hat{q}_{\sigma)},} \\
& {\left[P_{32}^{\varphi \omega}\left(1^{-}\right)\right]_{\rho \sigma}{ }^{\alpha \beta \gamma}=2 \hat{q}^{\alpha} L_{(\rho}^{[\beta} T_{\sigma)}^{\gamma]} \text {, }} \\
& {\left[P_{33}^{\varphi \varphi}\left(1^{-}\right)\right]_{\rho \sigma}{ }^{\alpha \beta}=2 T_{(\rho}^{(\alpha} L_{\sigma)}^{\beta)},}
\end{aligned}
$$




$$
\begin{aligned}
& {\left[P\left(0^{+}\right)\right]=\left[\begin{array}{cccc}
{\left[P_{11}^{\omega \omega}\left(0^{+}\right)\right]_{\tau \rho \sigma}{ }^{\alpha \beta \gamma}} & {\left[P_{12}^{\omega \varphi}\left(0^{+}\right)\right]_{\tau \rho \sigma}{ }^{\alpha \beta}} & {\left[P_{13}^{\omega \varphi}\left(0^{+}\right)\right]_{\tau \rho \sigma}{ }^{\alpha \beta}} & {\left[P_{14}^{\omega \sigma}\left(0^{+}\right)\right]_{\tau \rho \sigma}} \\
{\left[P_{21}^{\varphi \omega}\left(0^{+}\right)\right]_{\rho \sigma} \alpha \beta \gamma} & {\left[P_{22}^{\varphi \varphi}\left(0^{+}\right)\right]_{\rho \sigma}{ }_{\alpha \beta}} & {\left[P_{23}^{\varphi \varphi}\left(0^{+}\right)\right]_{\rho \sigma} \alpha \beta} & {\left[P_{24}^{\varphi \sigma}\left(0^{+}\right)\right]_{\rho \sigma}} \\
{\left[P_{31}^{\varphi \omega}\left(0^{+}\right)\right]_{\rho \sigma}{ }_{\alpha \beta \gamma}} & {\left[P_{32}^{\varphi \varphi}\left(0^{+}\right)\right]_{\rho \sigma}{ }^{\alpha \beta}} & {\left[P_{33}^{\varphi \varphi}\left(0^{+}\right)\right]_{\rho \sigma}{ }_{\alpha \beta}} & {\left[P_{34}^{\varphi \sigma}\left(0^{+}\right)\right]_{\rho \sigma}} \\
{\left[P_{41}^{\sigma \omega}\left(0^{+}\right)\right]^{\alpha \beta \gamma}} & {\left[P_{42}^{\sigma \varphi}\left(0^{+}\right)\right]^{\alpha \beta}} & {\left[P_{43}^{\sigma \varphi}\left(0^{+}\right)\right]^{\alpha \beta}} & {\left[P_{44}^{\sigma \sigma}\left(0^{+}\right)\right]}
\end{array}\right],} \\
& {\left[P_{11}^{\omega \omega}\left(0^{+}\right)\right]_{\tau \rho \sigma}{ }^{\alpha \beta \gamma}=\frac{2}{3} T_{\tau[\rho} L_{\sigma]}^{[\gamma} T^{\beta] \alpha},} \\
& {\left[P_{12}^{\omega \varphi}\left(0^{+}\right)\right]_{\tau \rho \sigma}{ }^{\alpha \beta}=\frac{\sqrt{2}}{3} T^{\alpha \beta} T_{\tau[\rho} \hat{q}_{\sigma]},} \\
& {\left[P_{13}^{\omega \varphi}\left(0^{+}\right)\right]_{\tau \rho \sigma}{ }^{\alpha \beta}=\sqrt{\frac{2}{3}} L^{\alpha \beta} T_{\tau[\rho} \hat{q}_{\sigma]},} \\
& {\left[P_{14}^{\omega \sigma}\left(0^{+}\right)\right]_{\tau \rho \sigma}=\sqrt{\frac{2}{3}} \hat{q}_{[\sigma} T_{\rho] \tau},} \\
& {\left[P_{21}^{\varphi \omega}\left(0^{+}\right)\right]_{\rho \sigma}{ }^{\alpha \beta \gamma}=\frac{\sqrt{2}}{3} T_{\rho \sigma} T^{\alpha[\beta} \hat{q}^{\gamma]},} \\
& {\left[P_{22}^{\varphi \varphi}\left(0^{+}\right)\right]_{\rho \sigma}{ }^{\alpha \beta}=\frac{1}{3} T_{\rho \sigma} T^{\alpha \beta},} \\
& {\left[P_{23}^{\varphi \varphi}\left(0^{+}\right)\right]_{\rho \sigma}{ }^{\alpha \beta}=\frac{1}{\sqrt{3}} T_{\rho \sigma} L^{\alpha \beta},} \\
& {\left[P_{24}^{\varphi \sigma}\left(0^{+}\right)\right]_{\rho \sigma}=\frac{1}{\sqrt{3}} T_{\rho \sigma},} \\
& {\left[P_{31}^{\varphi \omega}\left(0^{+}\right)\right]_{\rho \sigma}{ }^{\alpha \beta \gamma}=\sqrt{\frac{2}{3}} L_{\rho \sigma} T^{\alpha[\beta} \hat{q}^{\gamma]},} \\
& {\left[P_{32}^{\varphi \varphi}\left(0^{+}\right)\right]_{\rho \sigma}{ }^{\alpha \beta}=\frac{1}{\sqrt{3}} L_{\rho \sigma} T^{\alpha \beta},} \\
& {\left[P_{33}^{\varphi \varphi}\left(0^{+}\right)\right]_{\rho \sigma}{ }^{\alpha \beta}=L_{\rho \sigma} L^{\alpha \beta},} \\
& {\left[P_{34}^{\varphi \sigma}\left(0^{+}\right)\right]_{\rho \sigma}=L_{\rho \sigma} \text {, }} \\
& {\left[P_{41}^{\sigma \omega}\left(0^{+}\right)\right]^{\alpha \beta \gamma}=\sqrt{\frac{2}{3}} \hat{q}^{[\gamma} T^{\beta] \alpha},} \\
& {\left[P_{42}^{\sigma \varphi}\left(0^{+}\right)\right]^{\alpha \beta}=\frac{1}{\sqrt{3}} T^{\alpha \beta},} \\
& {\left[P_{43}^{\sigma \varphi}\left(0^{+}\right)\right]^{\alpha \beta}=L^{\alpha \beta} \text {, }} \\
& {\left[P_{44}^{\sigma \sigma}\left(0^{+}\right)\right]=1 \text {, }} \\
& {\left[P^{\omega \omega}\left(0^{-}\right)\right]_{\tau \rho \sigma}{ }^{\alpha \beta \gamma}=\frac{1}{3} T_{\tau}^{\alpha} T_{[\rho}^{[\beta} T_{\sigma]}^{\gamma]}-\frac{2}{3} T_{\tau}^{[\gamma} T_{[\rho}^{\beta]} T_{\sigma]}^{\alpha} \equiv T_{[\tau}^{[\alpha} T_{\rho}^{\beta} T_{\sigma]}^{\gamma]},}
\end{aligned}
$$




\section{References}

1. S. Weinberg, in "General Relativity: an Einstein Centenary Survey", ed. S. Hawking and W. Israel, Cambridge University Press (1986);

J.F. Donoghue, "General relativity as an effective field theory: the leading quantum correction", Univ. Mass. UMHEP-408 (gr-qc/9405057).

2. A.D. Sakharov, Dokl. Akad. Nauk, SSR 177, 70 (1967);

K. Akama, Y. Chikashige, T. Matsuki and H. Terazawa, Progr. Theor. Phys. 60, 868 (1978);

B. Hasslacher and E. Mottola, Phys. Lett. 95 B, 237 (1980);

S. Adler, Rev. Mod. Phys. 54, 729 (1982);

D. Amati and G. Veneziano, Nucl. Phys. B 204, 451 (1982);

I.L. Buchbinder and S.D. Odintsov, Class and Quantum Grav. 2, 721 (1985);

H. Terazawa, in the A.D. Sakharov memorial volume, ed. L.V. Keldysh et al., Nauka, Moscow (1991);

S.D. Odintsov and I.L. Shapiro, Class. and Quantum Grav. 9, (1992).

3. J. Gasser and H. Leutwyler, Ann. of Phys. (NY) 158, 142 (1984);

H. Leutwyler, Ann. of Phys. (NY) 235, 165 (1994).

4. K.S. Stelle, Phys. Rev. D 16, 953 (1977).

5. I.L. Buchbinder, S.D. Odintsov and I.L. Shapiro, Effective Action in Quantum Gravity, (IOP, Bristol (UK), 1992).

6. R. Utiyama, Phys. Rev. 101, 1597 (1956); Progr. Theor. Phys. 64, 2207 (1980);

T.W.B. Kibble, J. Math. Phys. 2, 212 (1961);

D.W. Sciama, in "Recent developments in General Relativity", Infeld Festschrift, Pergamon, Oxford (1962);

F.W. Hehl, P. von der Heyde, G.D. Kerlick and J.M. Nester, Rev. Mod. Phys. 48, 393 (1976);

L.L. Smalley, Phys. Lett. 61 A, 436 (1977);

K. Hayashi and Y. Shirafuji, Prog. Theor. Phys. 64, 866, 883, 1435, 2222 (1980);

D. Ivanenko and G. Sardanashvily, Phys. Rep. 94, 1 (1983).

7. R. Percacci, Phys. Lett. 144 B, 37 (1984); Nucl. Phys. B 353, 271 (1991).

8. E. Sezgin and P. van Nieuwenhuizen, Phys. Rev. D 21, 3269 (1980);

E. Sezgin, Phys. Rev. D 24, 1677 (1981);

R. Kuhfuss and J. Nitsch, Gen. Rel. and Grav. 18, 947 (1986).

9. N. Nakanishi and I. Ojima, Covariant operator formalism of gauge theory and quantum gravity, (World Scientific, Singapore, 1990).

10. R. Floreanini and R. Percacci, "Average effective potential for the conformal factor", Nucl. Phys. B, to appear.

11. R. Floreanini and R. Percacci, "A multiplicative background field method", in the volume in honor of D. Ivanenko, V. Koloskov, ed., to appear.

12. K.J. Barnes, Ph.D. Thesis (1963) unpublished;

R.J. Rivers, Nuovo Cim. 34387 (1964). 
13. K.G. Wilson and J.B. Kogut, Phys. Rep. 12C, 75 (1974);

K.G. Wilson, Rev. Mod. Phys. 47, 774 (1975).

14. J. Polchinski, Nucl. Phys. B 231, 269 (1984).

15. B. Warr, Ann. of Phys (NY) 183, 1 (1988)

C. Becchi, "On the construction of renormalized quantum field theory using renormalization group techniques", in Elementary Particles, Field Theory and Statistical Mechanics, M. Bonini, G. Marchesini and E. Onofri, eds., University of Parma (1993); M. Bonini, M. D'Attanasio and G. Marchesini, Nucl. Phys. B 418, 81 (1994); ibid. B 421, 429 (1994); "BRS symmetry for Yang-Mills theory with exact renormalization group", Parma Preprint, UPRF 94-412 (1994).

16. C. Wetterich, Nucl. Phys. B 334, 506 (1990); ibid. B 352, 529 (1991); Z. Phys. C 57, 451 (1993); ibid. C 60, 461 (1993);

M. Reuter and C. Wetterich, Nucl. Phys. B 391, 147 (1993).

17. M. Reuter and C. Wetterich, Nucl. Phys. B 417, 181 (1994); ibid. B 427, 291 (1994);

18. C. Ford, D.R.T. Jones, P.W. Stephenson and M.B. Einhorn, Nucl. Phys. B 395, 17 (1993).

19. F. Wegner and A. Houghton, Phys. Rev. A 8, 401 (1973);

A. Hasenfratz and P. Hasenfratz, Nucl. Phys. B 270, 685 (1986);

C. Wetterich, Phys. Lett. B 301, 90 (1993);

S.B. Liao and J. Polonyi, "Renormalization group and universality", Duke TH-94-64.

20. J. Julve and M. Tonin, Nuovo Cimento 46 B, 137 (1978);

A. Salam and J. Strathdee, Phys. Rev. D 18, 4480 (1978).

21. L. Griguolo and R. Percacci, in preparation.

22. N.C. Tsamis and R.P. Woodard, Ann. of Phys. 168, 457 (1986);

E.T. Tomboulis, Nucl. Phys. B 329, 410 (1990).

23. P. Baekler, F.W. Hehl and H.J. Lenzen, in Proceedings of the third Marcel Grossmann meeting on General Relativity, H. Ning, ed., (North Holland, Amsterdam, 1983). 